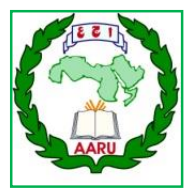

Arab Univ. J. Agric. Sci., Ain Shams Univ., Cairo, Egypt

28(2), 485-503, 2020

Website: http://ajs.journals.ekb.eg

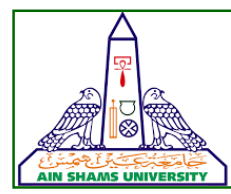

485

\title{
ALLEVIATION OF SALINITY STRESS THROUGH MAGNETIC WATER AND NANO ZINC AND MAGNESIUM TREATMENT OF LEMONGRASS PLANT (Cymbopogen citratus L.)
}

\author{
Abdel-Hamid* A.N. \\ Horticulture Dept., Fac. of Agric., Ain Shams Univ., P.O. Box 68, Hadayek Shoubra 11241, \\ Cairo, Egypt \\ *Corresponding author: nazmy604@yahoo.com
}

Received 15 July, 2020

Accepted 4 August, 2020

\section{ABSTRACT}

The effect of magnetic water compared with saline water, nano-Mg at 100 and 200 ppm and nano$\mathrm{Zn}$ at 50 and $100 \mathrm{ppm}$ treatments and their combinations on lemongrass plants (Cymbopogen citratus L.) was studied during 2018/2019 and 2019/2020.

Vegetative growth parameters including plant height, number of tillers/ plant, leaf area, herb fresh and dry weights were greatly affected by both studied factors. Magnetic water treatments recorded the highest values of all vegetative growth parameters compared with saline water. However, all nanoparticles of $\mathrm{Mg}$ and $\mathrm{Zn}$ treatments were superior than control in promoting vegetative growth parameters and the treatments of 100 ppm nano-Zn and 200 ppm nano-Mg recorded the maximum values of vegetative growth parameters. The combined treatments showed that the highest values of vegetative growth measurements were obtained in plants irrigated with magnetic water and sprayed with 100 ppm nano-Zn, in both cuts $\ln 1^{\text {st }}$ and $2^{\text {nd }}$ seasons.

$\mathrm{N}, \mathrm{P}, \mathrm{K}$ and $\mathrm{Mg}$ contents were significantly higher in lemongrass plants irrigated with magnetic water than those irrigated with saline water. Moreover, the treatments of $100 \mathrm{ppm}$ nano-Zn exhibited the highest significant values of $N, P, K$, whereas 100 and 200 ppm of nano-Mg treatments were superior in recording the highest values of $\mathrm{Mg}$ content, in the two seasons. The combination treatments of lemongrass plants irrigated with magnetic water and sprayed with 100 ppm nano-Zn or 200 ppm nano$\mathrm{Mg}$ produced the richest values of $\mathrm{N}, \mathrm{P}$ and $\mathrm{K} \%$ D.W., whereas, the treatments of magnetic water
\end{abstract}

combined with 100 or 200 ppm of nano-Mg produced the best Mg content.

A great effect of magnetic water irrigation on increasing $\mathrm{Fe}$ and $\mathrm{Zn}$ contents than saline water treatments. The treatments of 50 and 100 ppm of nano$\mathrm{Zn}$ were more effective than control and other treatments in maximizing $\mathrm{Fe}$ and $\mathrm{Zn}$ levels.

The combined treatments of lemongrass plants irrigated with magnetic water and sprayed with 100 ppm of nano-Zn exhibited the highest values of $\mathrm{Fe}$ and $\mathrm{Zn} \%$ D.W. in most cases. Additionally, total chlorophyll greatly increased in lemongrass plants irrigated with magnetic water than those irrigated with saline water, whereas the treatment of $200 \mathrm{ppm}$ nano-Mg increased it than others. Interaction effect showed that, plants irrigated with magnetic water and sprayed with nano-Mg at $200 \mathrm{ppm}$ exhibited the highest values of total chlorophyll.

Proline and glycine betaine contents were greatly decreased with magnetic water treatment compared with saline water. Meanwhile, all applied treatments were effective in decreasing the levels of both constituents than control, which considered a good indicator to alleviation of water salinity stress on lemongrass plants through magnetic water and nanoparticles of $\mathrm{Mg}$ and $\mathrm{Zn}$ applications. Finally, volatile oil \%, was greatly increased in lemongrass plants irrigated with magnetic water, especially the treatment of nano-Zn at 100 ppm as compared of others in this respect in both cuts and the two studied seasons. The interaction between the two studied factors was significant which means that both factors act together in improving volatile oil content in lemongrass herb. 
Keywords: Lemongrass, Magnetic Water, Saline water, Nanoparticles, Volatile oils, Proline and glycine betaine

\section{INTRODUCTION}

Lemongrass plant (Cymbopogon citratus L.) is the important individuals from Cymbopogon, is a basic fragrant cum therapeutic spice. It has a place with the family Poaceae and Cymbopogon genus, which comprise of extra than eighty species. Cymbopogon citratus L., is one of the fundamental oil crops productivity, created in various tropical countries in South America, Asia and Africa (Bagaturiya, 1990), it is turfed enduring grass with different solidified stems rising up out of a short, rhizomatous rootstock. The leaves are about one meter long and $3 \mathrm{~cm}$ wide and used as a high source of cellulose and paper creation (Ciaramello, 1973) Production of volatile oil of the stems and leaves ranged from 0.25 to $0.35 \%$, utilized for its lemon flavor and smell. It is used in cooking as a critical wellspring of lemon improving. Restoratively, it is quieting, antidiabetic, anthelmintic, antibacterial, antifungal, anticancer, antiplatelet, hepatoprotective, opiate and vasorelaxant. The unstable oil is carminative, depressant, torment easing, antipyretic (AbeSato et al 2002.Tiwari et al 2010 and Tyagi and Malik 2012)

Saltiness is the main abiotic stress factor, it confines the food creation and break down the developing interest of food crops. Saltiness is the significant worry of applied researchers to achieve the food gap around the world. Since, greater part of harvest plants species have a place with glycophyte class which they are vulnerable to salt pressure, thus it is the most basic natural pressure that can handicap crop efficiency around the world (Flowers. 2004 and Munns and Tester 2008). Saltiness stress causes negative effects on different biochemical and physiological procedures which are related with plant development and yield as photosynthesis, protein amalgamation and lipid digestion systems which in this manner are seriously influenced by saltiness disorder inside a plant (Parida and Das 2005). Plant development is truly influenced by salt pressure, and plants adjust to this abiotic condition, Through embracing a modificated systems (Jha et al 2010 and Shabala and Munns, 2012). Overabundance of salt particles in either water or soil causes huge changes in morphological, physiological and biochemical properties of plants, it incites osmotic disturbance in plants, decrease in photo- synthetic pathway, indistinct of colors, and unevenness of water assimilation and ions take-up. Salt harm is reliant on various factors, for example, cultivars, development stage, plant feeding types and other natural variables (Fatma et al 2016 and Chen et al 2017).

Magnetically treated water (MTW) is water which has been gone through a magnetic field before use by the plant. There are great advantages to utilizing such rewarded water, in spite of the fact that there is as yet extensive discussion with regards to its productivity. In this regard Deng and Feng, (2007) examined the component of polarization of water and proposed a hypothesis dependent on the sub-atomic structure of water. In the system proposed by Pang and Deng, (2008), the communication of the remotely applied attractive field with the electric momentum emerging from the protons (or hydrogen particles) upgrade conductivity along the shut hydrogen reinforced chains of atoms happening in water.

MTW expands the capacity of soil to trade particles and subsequently better absorption of supplements and mineral by plants. Utilization of MTW pulls in uncommon consideration because of its absence of contamination, wellbeing and effortlessness and it might assume a significant position in the rundown of naturally clean strategies (Bogatin, 1999). It might likewise be utilized to enhance water use efficiency because of its impacts on some physical and chemical properties of both the water and the soil (Noran et al 1996 and Flores et al 2007). MTW improved seed germination and seedling development of an assortment of many crops in saline calcareous soils (Hilal and Hillal, 2000), it additionally expanded tallness, size and number of leaves of maize seedlings (Flores et al 2007), sunflower (Maitwijczuk et al 2012) and wheat and vegetables (Waleed et al 2013)

Ongoing investigations demonstrated that nanoparticles initiate an advantageous impact on plant development and improvement, and control plant disorgers (Roghayyeh et al 2010 and Nair et al 2010). Nano fertilizers are utilized as of late as an option in contrast to traditional sources because of moderate discharge and high effective use by plants. Use of nano fertilizers are among the most encouraging strategies, which can possibly improve plant use effectiveness and decrease natural poisonousness. Plant use considerably less sum concoction chemical and pesticides than the sum applied in the soil, in this manner, rest of the synthetic substances stay unused and collect in soil to build soil harmfulness. The utilization of nano chemical 

magnesium Treatment of lemongrass plant (Cymbopogen citratus $L$.)

fertilizers could be an expected way to deal with address such issues of soil harmfulness and other related pressure issues. (Yang et al 2007 and Disfani et al 2017).

$\mathrm{ZnO}$ nano particles (nano-ZnO) have been generally applied to plants because of their exceptional physiochemical properties and huge explicit surface area (Xu et al 2009 and Yang et al 2019). Nanomaterials control plant development and improvement at various levels (Kwak and An 2016). For example, the advantageous impact of nano-ZnO at low fixations has been very much recorded in peanut (Prasad et al 2012), mung bean (Mahajan et al 2011), and tomato (Singh, et al 2016), proposing that nano particles create the resilience to different abiotic stresses, including dry season (Zaimenko et al 2014), saltiness (Almutairi, 2016) and low temperature (Hawrylak-Nowak et al 2010). The mitigation of abiotic disorder by nano particles was regularly connected with the improved cancer prevention agent catalysts exercises (Sturikova et al 2018).

Magnesium (Mg) is essential macro element for the chlorophyll synthesis and contributes up to $10 \%$ of the total $\mathrm{Mg}$ in the chlorophyll structure (Wilkinsan et al 1990). Mg2+ is involved in several vital processes of plants including the formation of ATP in chloroplasts, $\mathrm{CO}_{2}$ stabilization, protein synthesis, chlorophyll formation, development of phloem, and optical oxidation in leaves, it activates a large number of enzymes such as vas ATP-as enzymes, RuBP, RNA polymerases, and protein kinase (Cakmak and Yazici, 2010). However, Kumar et al (2006) emphasized that chlorophyll and carotenoids in rice plant decrease due to $\mathrm{Mg}$ deficiency while an increase in $\mathrm{Mg}$ in wheat plant induced more chlorophyll accumulation (El-Metwally et al 2010). $\mathrm{Mg}$ is a critical element which is required to maintain a high $\mathrm{pH}$ level in chloroplasts and cytoplasm. According to Wang et al (2004), the total amount of protein and soluble sugars in plant leaves decreased under the $\mathrm{Mg}$ insufficiency condition, and proline content. (Lesko et al 2002).

The target of present work is to evaluate the impact of magnetically treated water, nanoparticles of $\mathrm{Mg}$ and $\mathrm{Zn}$ and their interaction to minimize or alleviate salinity stress disorders through their effect on vegetative growth, chemical constituents and volatile oil content of lemongrass (Cymbopogon citratus L.) plant.

\section{MATERIAL AND METHODS}

This work was carried out during two successive seasons of 2018/2019 and 2019/2020 in Wadi ElNatron region, Behira Governorate to evaluate the effect of irrigation with magnetic water and spraying of magnesium and zinc nanoparticles on Cymbopogen citratus $L$. plant. The experiment included ten treatments, two irrigation water treatments (saline and magnetic water) and five nanoparticles treatments (two nano- levels $\mathrm{Mg}$ at 100 , $200 \mathrm{ppm}$ and two nano-levels $\mathrm{Zn}$ at 50, and 100 ppm, in addition to control).

Lemongrass plants were propagated vegetative through slips obtained by the splitting up of individual adult clumps. The rooted slips were cultivated on first week of April during the two successive seasons at spacing of $50 \mathrm{~cm}$ between hills and $100 \mathrm{~cm}$ between rows under drip irrigation system with 4 liters/hr. drippers for an hour three times in week. The chemical analysis of the used water (saline water) is illustrated in the following table:

\begin{tabular}{|c|c|c|c|c|c|c|c|c|c|}
\hline \multirow{2}{*}{$\mathbf{p H}$} & \multirow{2}{*}{$\mathbf{p p m}$} & \multicolumn{4}{|c|}{ Soluble anions meq/L } & \multicolumn{4}{c|}{ Soluble cations meq/L } \\
\cline { 3 - 10 } & & $\mathbf{C O}_{3}^{--}$ & $\mathbf{H C O}_{3}^{-}$ & $\mathbf{C l}^{-}$ & $\mathbf{C O}_{4}^{--}$ & $\mathbf{C a}^{++}$ & $\mathbf{M g}^{++}$ & $\mathbf{N a}^{+}$ & $\mathbf{K}^{+}$ \\
\hline 7.8 & 2820 & 0.2 & 1.96 & 21.5 & 16.08 & 9.60 & 7.45 & 21.25 & 1.44 \\
\hline
\end{tabular}

Regarding to magnetic water, it was obtained by magnetic field apparatus where irrigation water passed through a magnetic device which comprised of two magnets arranged to the north and south poles and installed on the main irrigation line before application to the lemongrass plants. The ten treatments were arranged in split plot design with four replicates were the two types of irrigation water were designed as in the main plots and the foliar spraying of nanoparticles treatments were randomly arranged in the sub-plots. All normal horticulture practices were done as usual in this respect.

\section{The used treatments were as follows}

1. Control irrigated with saline water

2. Control irrigated with magnetic water

3. Nano Mg at $100 \mathrm{ppm}$ irrigated with saline water. 
4. Nano $\mathrm{Mg}$ at $100 \mathrm{ppm}$ irrigated with magnetic water.

5. Nano $\mathrm{Mg}$ at $200 \mathrm{ppm}$ irrigated with saline water

6. Nano $\mathrm{Mg}$ at $200 \mathrm{ppm}$ irrigated with magnetic water.

7. Nano $\mathrm{Zn}$ at $50 \mathrm{ppm}$ irrigated with saline water.

8. Nano $\mathrm{Zn}$ at $50 \mathrm{ppm}$ irrigated with magnetic water.

9. Nano $\mathrm{Zn}$ at $100 \mathrm{ppm}$ irrigated with saline water.

10. Nano $\mathrm{Zn}$ at $100 \mathrm{ppm}$ irrigated with magnetic water.

The foliar sprays of Nano $\mathrm{Zn}$ and Nano Mg were carried out three times namely, at mid of May, mid of June and mid of July in both studied seasons and first cut was done in mid of November. However, the second stage of spraying was repeated at first of December and again second in $1^{\text {st }}$ of January in both seasons and second cut was adopted at first of February in both studied seasons. The following data were recorded at the two cuts:

\section{1- Vegetative growth parameters}

Plant height $(\mathrm{cm})$, number of tillers/plant, leaf area $\left(\mathrm{cm}^{2}\right)$, herb fresh weight/ plant $(\mathrm{g})$ and herb dry weight/ plant $(\mathrm{g})$.

\section{2- Chemical constituents}

\section{2-1- Mineral contents}

The herb of lemongrass plants were collected washed and dried at $70^{\circ} \mathrm{C}$ until a constant weight and then ground for nutrient elements determination. Nitrogen was measured according to Guebel et al (1991), phosphorus was measured according to Bringham (1982), potassium was measured according to Westerman (1990) and magnesium was determined according to Pohl et al (2016). Data of macroelements were expressed as $\mathrm{g} / 100 \mathrm{~g}$ dry weight (\%). However, Fe and $\mathrm{Zn}$ contents were determined according to Chapman and Pratt (1982).

\section{2-2 Total chlorophyll (meter reading)}

It was measured as SPAD units using Minolta chlorophyll meter (model SPAD $\mathrm{SO}_{2}$ ) according to AOAC (1990)

2-3 Proline amino acid content ( $\mu \mathrm{g} / \mathrm{g}$ dry weight) was determined in lemongrass herb according to Bates et al (1973).
2-4 Glycine betaine content ( $\mu \mathrm{g} / \mathrm{g}$ dry weight) was determined according to Escalante-Magana et al (2019).

2-5 Volatile oil \% was determined in the air dried herb by hydrodistillation for 3 hours using a Clevenger apparatus. The volatile oil (\%) was calculated as a relative percentage ( $\mathrm{v} / \mathrm{w})$ according to British Pharmacopoeia (1963)

\section{Statistical analysis}

The obtained data were statistically analyzed and the differences between the means of different treatments and their interactions subjected to analysis of variance (ANOVA) as factorial experiments in split plot design described by Snedecor and Cochran (1990) extracted from Waller and Duncan (1969).

\section{RESULTS AND DISCUSSION}

\section{1- Vegetative growth parameters}

\section{1-1- Plant height $(\mathrm{cm})$}

Data in Table (1) show the effect of magnetic water and nano materials of $\mathrm{Mg}$ and $\mathrm{Zn}$ treatments on plant height $(\mathrm{cm})$ of lemongrass (Cymbopogen citratus L.) during 2018/2019 and 2019/2020 seasons.

Magnetic water was superior in the two cuts and the two studied seasons than normal irrigation water (saline water) in increasing plant height with significant differences between them. However, nano-Zn at $100 \mathrm{ppm}$ and nano-Mg at $200 \mathrm{ppm}$ were effective than the other treatments or the control in improving plants height of lemongrass plants. The combined treatments gave the tallest plants were recorded in lemongrass plants irrigated with magnetic water and sprayed with $100 \mathrm{ppm}$ of nano-Zn. Generally, it could be noticed that plant height values were higher in second cut than in first one, irrespective of the used treatments.

Lemongrass plant is the elite member of the genus Cymbopogon cultivated for an essential aromatic cum medicinal herb (Bagaturiya, 1990). Nano-Zn application became usual for fertilization of horticulture fruits for many favorable benefits specially peerless physical properties and big specific area (Xu et al 2009 \& Yang et al 2019). 

magnesium Treatment of lemongrass plant (Cymbopogen citratus $L$.)

Table 1. Effect of magnetic water, nanoparticles of $\mathrm{Mg}$ and $\mathrm{Zn}$ and their interactions on plant height $(\mathrm{cm})$ of lemongrass (Cymbopogon citratus L.) plant, during 2018/2019 and 2019/2020 seasons

\begin{tabular}{|c|c|c|c|c|c|c|}
\hline \multirow{3}{*}{ Treatments } & \multicolumn{6}{|c|}{$\begin{array}{l}\text { Plant height (cm) } \\
2018 / 2019 \text { Season }\end{array}$} \\
\hline & \multicolumn{2}{|c|}{$1^{\text {st }}$ cut } & \multirow[b]{2}{*}{ Mean } & \multicolumn{2}{|c|}{$2^{\text {nd }}$ cut } & \multirow[b]{2}{*}{ Mean } \\
\hline & Saline water & $\begin{array}{l}\text { Magnetic } \\
\text { water }\end{array}$ & & Saline water & $\begin{array}{l}\text { Magnetic } \\
\text { water }\end{array}$ & \\
\hline Control & $44.7 \mathrm{~g}$ & $51.4 \mathrm{efg}$ & 48.1 D & $62.0 \mathrm{~h}$ & $65.0 \mathrm{f}$ & 63.5 D \\
\hline Nano Mg 100 ppm & 53.2 ef & $65.8 \mathrm{bc}$ & $59.5 \mathrm{C}$ & $64.0 \mathrm{~h}$ & $81.0 \mathrm{~d}$ & $72.5 \mathrm{C}$ \\
\hline Nano Mg 200 ppm & $61.3 \mathrm{~cd}$ & $70.8 \mathrm{ab}$ & $66.1 \mathrm{AB}$ & $66.0 \mathrm{~g}$ & $88.1 \mathrm{~b}$ & 77.1 B \\
\hline Nano Zn 50 ppm & $57.1 \mathrm{de}$ & $68.2 a b$ & $62.8 \mathrm{BC}$ & $65.0 \mathrm{~g}$ & $84.9 \mathrm{c}$ & $74.5 \mathrm{BC}$ \\
\hline Nano Zn 100 ppm & $64.3 \mathrm{bc}$ & $75.3 \mathrm{a}$ & $69.8 \mathrm{~A}$ & $74.3 \mathrm{e}$ & $91.5 \mathrm{a}$ & $82.9 \mathrm{~A}$ \\
\hline \multirow[t]{2}{*}{ Mean } & $56.1 \mathrm{~B}$ & $66.3 \mathrm{~A}$ & & $65.9 \mathrm{~B}$ & $82.1 \mathrm{~A}$ & \\
\hline & \multicolumn{6}{|c|}{ 2019/2020 Season } \\
\hline Nano Mg 100 ppm & $40.2 \mathrm{f}$ & $61.5 \mathrm{~cd}$ & $50.1 \mathrm{D}$ & $61.6 \mathrm{~h}$ & 68.6 ef & 65.1 D \\
\hline Nano Mg 200 ppm & $48.4 \mathrm{e}$ & $66.5 \mathrm{bc}$ & $57.5 \mathrm{C}$ & $63.3 \mathrm{gh}$ & $69.6 \mathrm{~d}$ & $66.5 \mathrm{CD}$ \\
\hline Nano Zn 50 ppm & $60.2 \mathrm{~cd}$ & $68.9 \mathrm{ab}$ & $64.6 \mathrm{~B}$ & $67.3 \mathrm{e}$ & $75.3 \mathrm{~b}$ & 71.3 AB \\
\hline Nano Zn 100 ppm & $56.9 d$ & $62.6 \mathrm{bcd}$ & $59.7 \mathrm{C}$ & $64.6 \mathrm{fg}$ & $71.6 \mathrm{c}$ & 68.1BC \\
\hline Nano Mg 100 ppm & $63.1 \mathrm{bc}$ & $73.8 \mathrm{a}$ & $68.5 \mathrm{~A}$ & $69.6 \mathrm{~d}$ & $78.0 \mathrm{a}$ & $73.8 \mathrm{~A}$ \\
\hline Mean & $53.8 \mathrm{~B}$ & $66.7 \mathrm{~A}$ & & $62.3 \mathrm{~B}$ & $72.6 \mathrm{~A}$ & \\
\hline
\end{tabular}

Values followed by the same letter (s) are not significantly different at $5 \%$ level

Magnetically water treatment reduces the bond angle of the hydrogen-oxygen within the water molecule, so, these formatting smaller clusters of water molecule than in ordinary water and then it is leads to enhanced absorption of water into the cell (Verma, 2011). It is well known that $\mathrm{Zn}$ involved in the structural of phosphorus components and mobilizing of some enzymes such as phytase and phosphatase. So, application of nano-Zn promoting the growth of plants through mobilization of phosphorus from inactive to active form (Tarafdar and Claassen 2003).

\section{1-2- Number of tillers/ plant}

A great effect to both studied factors on the number of tillers/ plant of lemongrass were recorded (Table 2). It is important to pointed out that increasing of number of tillers in lemongrass plant are considered a good finding due to increasing of fresh and dry weights, the economic parts in lemongrass plant. The higher values in this regards were obtained by magnetic water treatments in the two cuts of both seasons, with significant differences than saline water treatment. However, nano-Zn at 100 ppm was superior than other nano-treatments or control in recording the highest number of tillers/ plant. Interaction between the two studied factors was significant in most cases, the higher interaction values (36.5 and 51.3 tillers/ plant) were obtained with plants irrigated with magnetic water and sprayed with 100 ppm nano $\mathrm{Zn}$ in first and second cuts in first season only.

Recent studies showed that nano-particles induce a beneficial effect on plant growth and development (Roghayyeh et al 2010).

\section{1-3 Leaf area $\left(\mathrm{cm}^{3}\right)$}

It is clear from data in Table (3) that leaf area was greatly affected by both irrigation water quality and spraying of nano-Mg and $\mathrm{Zn}$ in in most cases. However, magnetic water treatment exhibited the largest leaves of lemongrass plant in the two cuts of both seasons, with clear significant effects between them. Saline water had a negative effect on leaf area, where it depressed the vegetative growth parameters which previously discussed and consequently it negatively affected average leaf area values. Moreover, nano $\mathrm{Mg}$ at $200 \mathrm{ppm}$ and nano-Zn at $100 \mathrm{ppm}$ recorded the maximum values of leaf area than other treatments or control in the cuts in both studied seasons. However, no significant differences were obtained between the two mentioned treatments in the first season in both cuts, but in second one nano-Zn at $100 \mathrm{ppm}$ was superior than 
Table 2. Effect of magnetic water, nanoparticles of $\mathrm{Mg}$ and $\mathrm{Zn}$ and their interactions on number of tillers /plant of lemongrass (Cymbopogon citratus L.) plant, during 2018/2019 and 2019/2020 seasons

\begin{tabular}{|c|c|c|c|c|c|c|}
\hline \multirow{3}{*}{ Treatments } & \multicolumn{6}{|c|}{$\begin{array}{l}\text { No. of tillers /plant } \\
2018 / 2019 \text { Season }\end{array}$} \\
\hline & \multicolumn{2}{|c|}{$1^{\text {st }}$ cut } & \multirow[b]{2}{*}{ Mean } & \multicolumn{2}{|c|}{$2^{\text {nd }}$ cut } & \multirow[b]{2}{*}{ Mean } \\
\hline & $\begin{array}{l}\text { Saline } \\
\text { water }\end{array}$ & $\begin{array}{c}\text { Magnetic } \\
\text { water }\end{array}$ & & $\begin{array}{r}\text { Saline } \\
\text { water }\end{array}$ & $\begin{array}{c}\text { Magnetic } \\
\text { water }\end{array}$ & \\
\hline Control & $20.4 \mathrm{e}$ & $23.7 \mathrm{de}$ & $22.1 \mathrm{D}$ & $22.8 \mathrm{~h}$ & $25.7 \mathrm{~g}$ & $24.3 \mathrm{~F}$ \\
\hline Nano Mg 100 ppm & $21.5 \mathrm{e}$ & $27.3 \mathrm{bcd}$ & $24.4 \mathrm{C}$ & $33.5 \mathrm{f}$ & 39.3 e & $36.4 \mathrm{D}$ \\
\hline Nano Mg 200 ppm & $24.6 \mathrm{de}$ & $32.1 \mathrm{ab}$ & $28.4 \mathrm{~B}$ & 40.7 de & $48.8 \mathrm{ab}$ & $44.8 \mathrm{~B}$ \\
\hline Nano Zn 50 ppm & $23.1 \mathrm{de}$ & 30.4 bc & $26.7 \mathrm{~B}$ & 38.4 e & $43.4 \mathrm{~cd}$ & $40.9 \mathrm{C}$ \\
\hline Nano Zn 100 ppm & $25.7 \mathrm{~cd}$ & $36.5 \mathrm{a}$ & $31.1 \mathrm{~A}$ & $44.6 \mathrm{bcd}$ & $51.3 \mathrm{a}$ & $48.0 \mathrm{~A}$ \\
\hline \multirow[t]{2}{*}{ Mean } & $23.1 \mathrm{~B}$ & $30.0 \mathrm{~A}$ & & $36.0 \mathrm{~B}$ & $41.7 \mathrm{~A}$ & \\
\hline & \multicolumn{6}{|c|}{ 2019/2020 season } \\
\hline Control & $18.2 \mathrm{e}$ & $21.7 \mathrm{de}$ & $20.0 \mathrm{D}$ & $22.4 \mathrm{f}$ & $27.1 \mathrm{ef}$ & $24.7 d$ \\
\hline Nano Mg 100 ppm & $20.8 \mathrm{de}$ & $24.8 \mathrm{~cd}$ & $22.8 \mathrm{C}$ & 26.5 ef & $31.7 \mathrm{~cd}$ & $29.1 \mathrm{C}$ \\
\hline Nano Mg 200 ppm & $23.3 \mathrm{~cd}$ & $30.5 a b$ & $26.9 \mathrm{~B}$ & $30.6 \mathrm{de}$ & $37.1 \mathrm{ab}$ & $33.9 \mathrm{~B}$ \\
\hline Nano Zn 50 ppm & $24.8 \mathrm{~cd}$ & $27.1 \mathrm{bc}$ & $26.0 \mathrm{~B}$ & 28.7 de & $37.4 \mathrm{bc}$ & $33.1 \mathrm{~B}$ \\
\hline Nano Zn 100 ppm & $25.4 \mathrm{bcd}$ & $33.4 \mathrm{a}$ & $29.4 \mathrm{~A}$ & $32.3 \mathrm{bcd}$ & $41.5 \mathrm{a}$ & $36.9 \mathrm{~A}$ \\
\hline Mean & $22.6 \mathrm{~B}$ & $27.5 \mathrm{~A}$ & & $28.1 \mathrm{~B}$ & $35.0 \mathrm{~A}$ & \\
\hline
\end{tabular}

Values followed by the same letter (s) are not significantly different at $5 \%$ level

Table 3. Effect of magnetic water, nanoparticles of $\mathrm{Mg}$ and $\mathrm{Zn}$ and their interactions on leaf area $\left(\mathrm{cm}^{2}\right)$ of lemongrass (Cymbopogon citratus L.) plant, during 2018/2019 and 2019/2020 seasons.

\begin{tabular}{|c|c|c|c|c|c|c|}
\hline \multirow{3}{*}{ Treatments } & \multicolumn{6}{|c|}{$\begin{array}{c}\text { Leaf area }\left(\mathrm{cm}^{2}\right) \\
2018 / 2019 \text { Season }\end{array}$} \\
\hline & \multicolumn{2}{|c|}{$1^{\text {st }}$ cut } & \multirow[b]{2}{*}{ Mean } & \multicolumn{2}{|c|}{$2^{\text {nd }}$ cut } & \multirow[b]{2}{*}{ Mean } \\
\hline & $\begin{array}{l}\text { Saline } \\
\text { water }\end{array}$ & $\begin{array}{l}\text { Magnetic } \\
\text { water }\end{array}$ & & $\begin{array}{l}\text { Saline } \\
\text { water }\end{array}$ & $\begin{array}{c}\text { Magnetic } \\
\text { water }\end{array}$ & \\
\hline Control & $37.2 \mathrm{f}$ & $43.1 \mathrm{de}$ & $40.2 \mathrm{C}$ & $45.6 \mathrm{~g}$ & $49.7 \mathrm{f}$ & $47.7 \mathrm{D}$ \\
\hline Nano Mg 100 ppm & 40.7 ef & $44.7 \mathrm{de}$ & $42.7 \mathrm{C}$ & $46.6 \mathrm{f}$ & $57.1 \mathrm{~cd}$ & $51.9 \mathrm{C}$ \\
\hline Nano Mg 200 ppm & $54.4 \mathrm{c}$ & $66.0 \mathrm{a}$ & $60.2 \mathrm{~A}$ & $53.2 \mathrm{de}$ & $68.2 \mathrm{a}$ & $60.7 \mathrm{~A}$ \\
\hline Nano Zn 50 ppm & $48.0 \mathrm{~d}$ & $58.3 \mathrm{bc}$ & $53.1 \mathrm{~B}$ & 51.4 ef & $64.3 \mathrm{ab}$ & $57.9 \mathrm{~B}$ \\
\hline Nano Zn 100 ppm & $55.4 \mathrm{c}$ & $62.6 \mathrm{ab}$ & $59.0 \mathrm{~A}$ & $61.3 \mathrm{bc}$ & $65.8 \mathrm{ab}$ & $63.4 \mathrm{~A}$ \\
\hline \multirow[t]{2}{*}{ Mean } & 47.1 B & $54.9 \mathrm{~A}$ & & $51.6 \mathrm{~B}$ & $61.0 \mathrm{~A}$ & \\
\hline & \multicolumn{6}{|c|}{ 2019/2020 Season } \\
\hline Control & $40.2 \mathrm{f}$ & 44.7 de & $42.5 \mathrm{C}$ & $40.9 \mathrm{~g}$ & $45.0 \mathrm{fg}$ & $43.0 \mathrm{D}$ \\
\hline Nano Mg 100 ppm & 41.9 ef & $48.4 \mathrm{~d}$ & $45.1 \mathrm{C}$ & 47.0 ef & 48.8 def & $47.9 \mathrm{C}$ \\
\hline Nano Mg 200 ppm & $46.2 \mathrm{~d}$ & $60.2 \mathrm{bc}$ & $53.2 \mathrm{~B}$ & $52.3 \mathrm{~cd}$ & $61.4 a b$ & $56.9 \mathrm{~B}$ \\
\hline Nano Zn 50 ppm & $44.4 \mathrm{e}$ & $62.6 \mathrm{a}$ & $53.5 \mathrm{~B}$ & $50.6 \mathrm{de}$ & $57.2 \mathrm{bc}$ & $53.9 \mathrm{~B}$ \\
\hline Nano Zn 100 ppm & $56.9 \mathrm{c}$ & $61.5 \mathrm{ab}$ & $59.2 \mathrm{~A}$ & $56.5 \mathrm{bc}$ & $63.9 \mathrm{a}$ & $60.2 \mathrm{~A}$ \\
\hline Mean & $45.9 \mathrm{~B}$ & $55.5 \mathrm{~A}$ & & $49.5 \mathrm{~B}$ & $61.3 \mathrm{~A}$ & \\
\hline
\end{tabular}

Values followed by the same letter (s) are not significantly different at $5 \%$ level 

magnesium Treatment of lemongrass plant (Cymbopogen citratus $L$.)

200 ppm of nano-Mg in this respect. However, interaction values showed that lemongrass plant irrigated with magnetic water and sprayed with 200 ppm nano-Mn produced the largest leaves in both cuts in the first season of study.

\section{1-4- Herb fresh weight (g/plant).}

Data illustrated in Table (4) showed that the fresh weight of lemongrass plant was greatly improved with the both studied factors during the two cuts and the two studied seasons. However, magnetic water was superior than normal irrigation water (saline water) in recording the highest values of herb fresh weight in the two cuts and in both studied seasons.

Nano-Mg and $\mathrm{Zn}$ treatments greatly improved herb fresh weights of lemongrass plants than control in all cases, the treatments of nano- $\mathrm{Zn}$ at 100 ppm was superior than others, followed by 50 ppm nano-Zn and 200 ppm nano-Mg ,whereas the treatment of 100 ppm of nano-Mg came later. The combination treatments were scored significant in most cases, where the higher interaction values were recorded by lemongrass plants irrigated with magnetic water and sprayed with 100 ppm of nano-Zn in both cuts and both studied seasons.

Overabundance of salt particles in either water or soil causes huge changes in morphological, physiological and biochemical properties of plants, it incites osmotic disturbance in plants, decrease in photosynthetic pathway, indistinct of colors, and unevenness of water assimilation and ions take-up. Salt harm is reliant on various factors, for example, cultivars, development stage, plant feeding types and other natural variables (Fatma et al 2016 and Chen et al 2017).

\section{1-5 Herb dry weight (g/plant)}

A similar trend to those found on herb fresh weight values was also obtained on herb dry weight of lemongrass plant (Table 5). The heaviest herb dry weights were recorded by lemongrass plants irrigated with magnetic water against saline water in both cuts and both studied seasons with significant differences between them in all cases. Regarding the effect of different treatments of nanoparticles of $\mathrm{Mg}$ and $\mathrm{Zn}$, it is clear that $100 \mathrm{ppm}$ of nano-Zn treatment was more effective than other treatments or control in producing high values of herb dry weights in both cuts and both seasons. No significant differences were recorded between 50 ppm nano- $\mathrm{Zn}$ and
200 ppm nano-Mg treatments concerning herb dry weights of lemongrass plant in all cases. Values of interaction between the two studied factors showed that the highest herb dry weights of lemongrass plants were recorded in both cuts and both seasons in plants irrigated with magnetic water and sprayed with 100 ppm nano-Zn.

Since, majority of major crop plants species belong to glycophyte category, they are susceptible to salt stress hence is most critical environmental stress that can cripple crop productivity worldwide (Flowers 2004 \& Munns and Tester 2008).

Nano-fertilizers are used recently as an alternative to conventional fertilizers due to slow release and efficient use by plants. Application of nano fertilizers are among the most promising method which can potentially enhance plant resource use efficiency and reduce environmental toxicity due to accumulation of unused chemical fertilizers and pesticides in the soil (Disfani et al 2017).

\section{2- Chemical constituents}

\section{2-1- N, P and K (\% D.W.)}

Data presented in Tables (6, 7 and 8) show the effect of magnetic irrigation water, nano-Mg and $\mathrm{Zn}$ and their interactions on N,P and K\% D.W. of lemongrass plant. The higher values of $\mathrm{N}, \mathrm{P}$ and $\mathrm{K} \%$ $D$.W. were recorded gained in lemongrass plants irrigated with magnetic water in both cuts and both studied seasons. However, all applied treatments of nano particles were effective in increasing the levels of $\mathrm{N}, \mathrm{P}$ and $\mathrm{K}$ compared to control, the treatment of 100 ppm nano-Zn was more pronounced treatments in positive effect in this respect. $\mathrm{N} \%$ was $1.66 \%$ in magnetic water treated plants against $1.38 \%$ in saline water irrigated plants, $\mathrm{P} \%$ was $0.25 \%$ against $0.21 \%$ and $\mathrm{K} \%$ recorded $1.55 \%$ composed to $1.37 \%$.

However, the highest N, P and K\% were $1.74 \%$, 0.26 and $1.81 \%$ with $100 \mathrm{ppm}$ of nano-Zn against $1.30 \%, 0.19 \%$ and $1.11 \%$ for control lemongrass plants which declare the great effect to nanoparticles of $\mathrm{Mg}$ and $\mathrm{Zn}$ application in improving the nutrition status of the treated plants. Interaction values were act together in significant effect in this regard, the highest interaction values of the three macro elements were recorded in lemongrass plants irrigated with magnetic water and sprayed with the 100 ppm of nano-Zn. Plant use considerably less sum concoction chemical and pesticides than the sum applied in the soil, in this manner, rest of the synthetic substances stay unused and collect in soil to 
Table 4. Effect of magnetic water, nanoparticles of $\mathrm{Mg}$ and $\mathrm{Zn}$ and their interactions on herb fresh weight / plant (g) of lemongrass (Cymbopogon citratus L.) plant, during 2018/2019 and 2019/2020 seasons.

\begin{tabular}{|c|c|c|c|c|c|c|}
\hline \multirow{3}{*}{ Treatments } & \multicolumn{6}{|c|}{$\begin{array}{l}\text { Herb fresh weight / plant }(g) \\
2018 / 2019 \text { Season }\end{array}$} \\
\hline & \multicolumn{2}{|c|}{$1^{\text {st }}$ cut } & \multirow[b]{2}{*}{ Mean } & \multicolumn{2}{|c|}{$2^{\text {nd }}$ cut } & \multirow[b]{2}{*}{ Mean } \\
\hline & $\begin{array}{l}\text { Saline } \\
\text { water }\end{array}$ & $\begin{array}{c}\text { Magnetic } \\
\text { water }\end{array}$ & & $\begin{array}{c}\text { Saline } \\
\text { water }\end{array}$ & $\begin{array}{c}\text { Magnetic } \\
\text { water }\end{array}$ & \\
\hline Control & $137.2 \mathrm{f}$ & $184.5 \mathrm{e}$ & $160.9 \mathrm{D}$ & $215.7 \mathrm{~g}$ & $252.2 \mathrm{f}$ & $234.0 \mathrm{D}$ \\
\hline Nano Mg 100 ppm & $174.1 \mathrm{e}$ & $225.8 \mathrm{~cd}$ & $200.0 \mathrm{C}$ & $235.5 \mathrm{fg}$ & $317.8 d$ & $276.7 \mathrm{C}$ \\
\hline Nano Mg 200 ppm & $206.7 d$ & $284.5 \mathrm{~b}$ & 245.6 B & $278.5 \mathrm{e}$ & $369.6 \mathrm{~b}$ & $274.1 \mathrm{C}$ \\
\hline Nano Zn 50 ppm & 212.8. d & $244.0 \mathrm{c}$ & $228.4 \mathrm{~B}$ & $267.0 \mathrm{e}$ & $347.6 \mathrm{c}$ & $307.3 \mathrm{~B}$ \\
\hline Nano Zn 100 ppm & $235.1 \mathrm{c}$ & $336.1 \mathrm{a}$ & $285.6 \mathrm{~A}$ & $332.1 \mathrm{c}$ & $394.9 \mathrm{a}$ & $363.5 \mathrm{~A}$ \\
\hline \multirow[t]{2}{*}{ Mean } & $193.2 \mathrm{~B}$ & $255.0 \mathrm{~A}$ & & 265.8 B & $316.4 \mathrm{~A}$ & \\
\hline & \multicolumn{6}{|c|}{ 2019/2020 Season } \\
\hline Control & $127.1 \mathrm{~g}$ & $155.5 \mathrm{f}$ & $141.3 \mathrm{E}$ & $189.3 \mathrm{G}$ & $247.5 \mathrm{e}$ & $218.4 \mathrm{D}$ \\
\hline Nano Mg 100 ppm & $154.8 f$ & $195.0 \mathrm{e}$ & $174.9 \mathrm{D}$ & $221.7 f$ & $265.1 \mathrm{de}$ & $243.4 \mathrm{C}$ \\
\hline Nano Mg 200 ppm & $185.7 \mathrm{e}$ & $295.0 \mathrm{~b}$ & $240.4 \mathrm{C}$ & $251.4 \mathrm{de}$ & $337.2 \mathrm{~b}$ & $294.3 \mathrm{~B}$ \\
\hline Nano Zn 50 ppm & $224.1 d$ & $242.7 \mathrm{~cd}$ & $233.4 \mathrm{~B}$ & $269.1 d$ & $327.2 \mathrm{bc}$ & 298.2 B \\
\hline Nano Zn 100 ppm & $252.2 \mathrm{c}$ & $345.0 \mathrm{a}$ & $298.6 \mathrm{~A}$ & $316.1 \mathrm{c}$ & $371.9 \mathrm{a}$ & $344.0 \mathrm{~A}$ \\
\hline Mean & $188.8 \mathrm{~B}$ & $246.6 \mathrm{~A}$ & & $249.5 B$ & $309.8 \mathrm{~A}$ & \\
\hline
\end{tabular}

Values followed by the same letter (s) are not significantly different at $5 \%$ level

Table 5. Effect of magnetic water, nanoparticles of $\mathrm{Mg}$ and $\mathrm{Zn}$ and their interactions on herb dry weight / plant (g) of lemongrass (Cymbopogon citratus L.) plant, during 2018/2019 and 2019/2020 seasons.

\begin{tabular}{|c|c|c|c|c|c|c|}
\hline \multirow{3}{*}{ Treatments } & \multicolumn{6}{|c|}{$\begin{array}{l}\text { Herb dry weight / plant (g) } \\
2018 / 2019 \text { Season }\end{array}$} \\
\hline & \multicolumn{2}{|c|}{$1^{\text {st }}$ cut } & \multirow[b]{2}{*}{ Mean } & \multicolumn{2}{|c|}{$2^{\text {nd }}$ cut } & \multirow[b]{2}{*}{ Mean } \\
\hline & $\begin{array}{l}\text { Saline } \\
\text { water }\end{array}$ & $\begin{array}{l}\text { Magnetic } \\
\text { water }\end{array}$ & & $\begin{array}{l}\text { Saline } \\
\text { water }\end{array}$ & $\begin{array}{l}\text { Magnetic } \\
\text { water }\end{array}$ & \\
\hline Control & $52.1 \mathrm{~g}$ & 71.1 ef & $61.6 \mathrm{D}$ & $82.1 \mathrm{~g}$ & 98.1 ef & $90.1 \mathrm{D}$ \\
\hline Nano Mg 100 ppm & $66.2 \mathrm{f}$ & $85.9 \mathrm{~cd}$ & $79.1 \mathrm{C}$ & $89.3 \mathrm{fg}$ & $123.4 \mathrm{c}$ & $106.4 \mathrm{C}$ \\
\hline Nano Mg 200 ppm & 75.6 ef & $106.3 \mathrm{~b}$ & $91.0 \mathrm{~B}$ & $109.2 d$ & $144.3 \mathrm{ab}$ & $123.5 \mathrm{~B}$ \\
\hline Nano Zn 50 ppm & $80.9 \mathrm{de}$ & $92.7 \mathrm{c}$ & $86.8 \mathrm{~B}$ & $102.6 \mathrm{de}$ & $137.5 b$ & $120.1 \mathrm{~B}$ \\
\hline Nano Zn 100 ppm & $89.4 \mathrm{~cd}$ & $131.9 \mathrm{a}$ & $110.7 \mathrm{~A}$ & $126.7 \mathrm{c}$ & $148.9 \mathrm{a}$ & $137.8 \mathrm{~A}$ \\
\hline \multirow[t]{2}{*}{ Mean } & $72.8 \mathrm{~B}$ & $97.6 \mathrm{~A}$ & & $102.0 \mathrm{~B}$ & $129.8 \mathrm{~A}$ & \\
\hline & \multicolumn{6}{|c|}{ 2019/2020 Season } \\
\hline Control & $48.6 \mathrm{~g}$ & $62.4 f$ & $55.5 \mathrm{D}$ & $72.2 \mathrm{f}$ & $97.2 \mathrm{c}$ & $84.7 \mathrm{D}$ \\
\hline Nano Mg 100 ppm & $62.4 \mathrm{f}$ & $76.0 \mathrm{e}$ & $69.2 \mathrm{C}$ & $85.5 d$ & $100.7 \mathrm{c}$ & $93.1 \mathrm{C}$ \\
\hline Nano Mg 200 ppm & 70.2 ef & $114.1 \mathrm{~b}$ & 92.2 B & $97.5 \mathrm{c}$ & $130.6 \mathrm{~b}$ & $114.1 \mathrm{~B}$ \\
\hline Nano Zn 50 ppm & $85.5 d$ & $93.1 \mathrm{~cd}$ & 89.3 B & $102.6 \mathrm{c}$ & $129.1 \mathrm{~b}$ & $115.9 \mathrm{~B}$ \\
\hline Nano Zn 100 ppm & $99.4 \mathrm{c}$ & $136.5 \mathrm{a}$ & $118.0 \mathrm{~A}$ & $123.2 \mathrm{~b}$ & $144.4 \mathrm{a}$ & $133.8 \mathrm{~A}$ \\
\hline Mean & $73.2 \mathrm{~B}$ & $96.4 \mathrm{~A}$ & & $96.2 \mathrm{~B}$ & $120.4 \mathrm{~A}$ & \\
\hline
\end{tabular}

Values followed by the same letter (s) are not significantly different at $5 \%$ level 

magnesium Treatment of lemongrass plant (Cymbopogen citratus $L$.)

Table 6. Effect of magnetic water, nanoparticles of $\mathrm{Mg}$ and $\mathrm{Zn}$ and their interactions on N\% D.W. of lemongrass (Cymbopogon citratus L.) plant, during 2018/2019 and 2019/2020 seasons.

\begin{tabular}{|c|c|c|c|c|c|c|}
\hline \multirow{3}{*}{ Treatments } & \multicolumn{6}{|c|}{$\begin{array}{c}\text { N\% (D.W.) } \\
\text { 2018/2019 Season }\end{array}$} \\
\hline & \multicolumn{2}{|c|}{$1^{\text {st }}$ cut } & \multirow[b]{2}{*}{ Mean } & \multicolumn{2}{|c|}{$2^{\text {nd }}$ cut } & \multirow[b]{2}{*}{ Mean } \\
\hline & $\begin{array}{l}\text { Saline } \\
\text { water }\end{array}$ & $\begin{array}{l}\text { Magnetic } \\
\text { water }\end{array}$ & & $\begin{array}{l}\text { Saline } \\
\text { water }\end{array}$ & $\begin{array}{l}\text { Magnetic } \\
\text { water }\end{array}$ & \\
\hline Control & $1.20 \mathrm{e}$ & $1.39 \mathrm{~cd}$ & $1.30 \mathrm{D}$ & $1.15 \mathrm{e}$ & $1.42 \mathrm{~d}$ & $1.29 \mathrm{D}$ \\
\hline Nano Mg 100 ppm & $1.26 \mathrm{de}$ & $1.55 \mathrm{bc}$ & $1.41 \mathrm{C}$ & $1.36 \mathrm{~d}$ & $1.67 \mathrm{c}$ & $1.52 \mathrm{C}$ \\
\hline Nano Mg 200 ppm & $1.42 \mathrm{~cd}$ & $1.83 \mathrm{a}$ & $1.63 \mathrm{~B}$ & $1.70 \mathrm{c}$ & $1.93 \mathrm{~b}$ & $1.82 \mathrm{~B}$ \\
\hline Nano Zn 50 ppm & $1.40 \mathrm{~cd}$ & $1.70 a b$ & $1.55 \mathrm{~B}$ & $1.69 \mathrm{c}$ & $1.82 \mathrm{bc}$ & $1.76 \mathrm{~B}$ \\
\hline Nano Zn 100 ppm & $1.63 \mathrm{~b}$ & $1.85 \mathrm{a}$ & $1.74 \mathrm{~A}$ & $1.81 \mathrm{bc}$ & $2.17 \mathrm{a}$ & $1.99 \mathrm{~A}$ \\
\hline \multirow[t]{2}{*}{ Mean } & $1.38 \mathrm{~B}$ & $1.66 \mathrm{~A}$ & & $1.54 \mathrm{~B}$ & $1.80 \mathrm{~A}$ & \\
\hline & \multicolumn{6}{|c|}{ 2019/2020 Season } \\
\hline Control & $1.18 \mathrm{e}$ & $1.46 \mathrm{~cd}$ & $1.32 \mathrm{D}$ & $1.21 \mathrm{e}$ & $1.35 \mathrm{de}$ & $1.28 \mathrm{D}$ \\
\hline Nano Mg 100 ppm & $1.39 \mathrm{~d}$ & $1.50 \mathrm{~cd}$ & $1.45 \mathrm{C}$ & $1.42 \mathrm{~cd}$ & $1.51 \mathrm{~cd}$ & $1.47 \mathrm{C}$ \\
\hline Nano Mg 200 ppm & $1.42 \mathrm{~d}$ & $1.84 \mathrm{a}$ & $1.63 \mathrm{~B}$ & $1.58 \mathrm{c}$ & $1.76 a b$ & $1.67 \mathrm{~B}$ \\
\hline Nano Zn 50 ppm & $1.42 \mathrm{~d}$ & $1.76 \mathrm{ab}$ & $1.59 \mathrm{~B}$ & $1.53 \mathrm{~cd}$ & $1.78 a b$ & $1.66 \mathrm{~B}$ \\
\hline Nano Zn 100 ppm & $1.63 \mathrm{bc}$ & $1.93 \mathrm{a}$ & $1.78 \mathrm{~A}$ & $1.77 \mathrm{~b}$ & $1.98 \mathrm{a}$ & $1.88 \mathrm{~A}$ \\
\hline Mean & $1.40 \mathrm{~B}$ & $1.70 \mathrm{~A}$ & & $1.50 \mathrm{~B}$ & $1.68 \mathrm{~A}$ & \\
\hline
\end{tabular}

Values followed by the same letter (s) are not significantly different at $5 \%$ level

Table 7. Effect of magnetic water, nanoparticles of $\mathrm{Mg}$ and $\mathrm{Zn}$ and their interactions on P\% D.W. of lemongrass (Cymbopogon citratus L.) plant, during 2018/2019 and 2019/2020 seasons.

\begin{tabular}{|c|c|c|c|c|c|c|}
\hline \multirow{3}{*}{ Treatments } & \multicolumn{6}{|c|}{$\begin{array}{c}\text { P\% ( D.W.) } \\
\text { 2018/2019 Season }\end{array}$} \\
\hline & \multicolumn{2}{|c|}{$1^{\text {st }}$ cut } & \multirow[b]{2}{*}{ Mean } & \multicolumn{2}{|c|}{$2^{\text {nd }}$ cut } & \multirow[b]{2}{*}{ Mean } \\
\hline & $\begin{array}{l}\text { Saline } \\
\text { water }\end{array}$ & $\begin{array}{c}\text { Magnetic } \\
\text { water }\end{array}$ & & $\begin{array}{l}\text { Saline } \\
\text { water }\end{array}$ & $\begin{array}{c}\text { Magnetic } \\
\text { water }\end{array}$ & \\
\hline Control & $0.17 \mathrm{c}$ & $0.21 b c$ & $0.19 \mathrm{C}$ & $0.19 \mathrm{c}$ & $0.21 \mathrm{c}$ & $0.20 \mathrm{C}$ \\
\hline Nano Mg 100 ppm & $0.20 \mathrm{bc}$ & $0.23 a b$ & $0.22 \mathrm{~B}$ & $0.22 \mathrm{bc}$ & $0.27 a b$ & $0.25 \mathrm{AB}$ \\
\hline Nano Mg 200 ppm & $0.20 \mathrm{bc}$ & $0.25 a$ & $023 \mathrm{AB}$ & $0.21 \mathrm{c}$ & $0.27 \mathrm{ab}$ & $0.24 \mathrm{~B}$ \\
\hline Nano Zn 50 ppm & $0.23 a b$ & $0.27 a$ & $0.25 \mathrm{AB}$ & $0.23 \mathrm{abc}$ & $0.28 \mathrm{a}$ & $0.26 \mathrm{AB}$ \\
\hline Nano Zn 100 ppm & $0.24 a b$ & $0.28 a$ & $0.26 \mathrm{~A}$ & $0.28 \mathrm{a}$ & $0.26 \mathrm{a}$ & $0.27 \mathrm{~A}$ \\
\hline \multirow[t]{2}{*}{ Mean } & $0.21 \mathrm{~B}$ & $0.25 \mathrm{~A}$ & & $0.23 \mathrm{~B}$ & $0.26 \mathrm{~A}$ & \\
\hline & \multicolumn{6}{|c|}{ 2019/2020 Season } \\
\hline Control & $0.17 \mathrm{c}$ & $0.22 \mathrm{bc}$ & $0.20 \mathrm{C}$ & $0.14 \mathrm{~d}$ & $0.18 \mathrm{~cd}$ & $0.16 \mathrm{C}$ \\
\hline Nano Mg 100 ppm & $0.23 b$ & $0.28 \mathrm{a}$ & $0.26 \mathrm{AB}$ & $0.22 b c$ & $0.25 \mathrm{ab}$ & $0.24 \mathrm{~B}$ \\
\hline 0.Nano Mg 200 ppm & $0.22 b$ & $0.27 a b$ & $0.25 \mathrm{~B}$ & $0.22 \mathrm{bc}$ & $0.28 \mathrm{a}$ & $0.25 \mathrm{AB}$ \\
\hline Nano Zn 50 ppm & $0.25 a b$ & $0.28 \mathrm{a}$ & $0.27 \mathrm{AB}$ & $0.23 \mathrm{bc}$ & $0.28 \mathrm{a}$ & $0.26 \mathrm{AB}$ \\
\hline Nano Zn 100 ppm & $0.27 \mathrm{ab}$ & $0.29 \mathrm{a}$ & $0.28 \mathrm{~A}$ & $0.27 \mathrm{ab}$ & $0.29 \mathrm{a}$ & $0.28 \mathrm{~A}$ \\
\hline Mean & $0.23 \mathrm{~B}$ & $0.27 \mathrm{~A}$ & & 0.22 B & $0.26 \mathrm{~A}$ & \\
\hline
\end{tabular}

Values followed by the same letter (s) are not significantly different at $5 \%$ level 
Table 8. Effect of magnetic water, nanoparticles of $\mathrm{Mg}$ and $\mathrm{Zn}$ and their interactions on $\mathrm{K} \% \mathrm{D}$.W. of lemongrass (Cymbopogon citratus L.) plant, during 2018/2019 and 2019/2020 seasons.

\begin{tabular}{|c|c|c|c|c|c|c|}
\hline \multirow{3}{*}{ Treatments } & \multicolumn{6}{|c|}{$\begin{array}{c}\text { K \% D.W. } \\
\text { 2018/2019 Season }\end{array}$} \\
\hline & \multicolumn{2}{|c|}{$1^{\text {st }}$ cut } & \multirow[b]{2}{*}{ Mean } & \multicolumn{2}{|c|}{$2^{\text {nd }}$ cut } & \multirow[b]{2}{*}{ Mean } \\
\hline & $\begin{array}{l}\text { Saline } \\
\text { water }\end{array}$ & $\begin{array}{l}\text { Magnetic } \\
\text { water }\end{array}$ & & $\begin{array}{l}\text { Saline } \\
\text { water }\end{array}$ & $\begin{array}{l}\text { Magnetic } \\
\text { water }\end{array}$ & \\
\hline Control & $1.08 \mathrm{f}$ & 1.14 ef & $1.11 \mathrm{D}$ & $1.19 \mathrm{e}$ & $1.32 \mathrm{de}$ & $1.26 \mathrm{C}$ \\
\hline Nano Mg 100 ppm & 1.12 ef & $1.32 \mathrm{de}$ & $1.22 \mathrm{C}$ & $1.33 \mathrm{de}$ & $1.61 \mathrm{~b}$ & $1.47 \mathrm{~B}$ \\
\hline Nano Mg 200 ppm & $1.42 \mathrm{~cd}$ & $1.78 a b$ & $1.60 \mathrm{~B}$ & $1.57 \mathrm{bc}$ & $1.70 a b$ & $1.64 \mathrm{~A}$ \\
\hline Nano Zn 50 ppm & $1.49 \mathrm{~cd}$ & $1.63 \mathrm{bc}$ & $1.64 \mathrm{~B}$ & $1.40 \mathrm{~cd}$ & $1.59 \mathrm{bc}$ & $1.50 \mathrm{~B}$ \\
\hline Nano Zn 100 ppm & $1.74 a b$ & $1.88 \mathrm{a}$ & $1.81 \mathrm{~A}$ & $1.51 \mathrm{bc}$ & $1.83 \mathrm{a}$ & $1.67 \mathrm{~A}$ \\
\hline \multirow[t]{2}{*}{ Mean } & 1.37 B & $1.55 \mathrm{~A}$ & & $1.40 \mathrm{~B}$ & $1.61 \mathrm{~A}$ & \\
\hline & \multicolumn{6}{|c|}{ 2019/2020 Season } \\
\hline Control & $1.10 \mathrm{~d}$ & $1.27 \mathrm{~cd}$ & $1.19 \mathrm{D}$ & $1.16 \mathrm{e}$ & $1.34 \mathrm{de}$ & $1.25 \mathrm{D}$ \\
\hline Nano Mg 100 ppm & $1.22 \mathrm{~cd}$ & $1.42 \mathrm{c}$ & $1.32 \mathrm{C}$ & $1.20 \mathrm{e}$ & $1.56 \mathrm{bc}$ & $1.38 \mathrm{C}$ \\
\hline Nano Mg 200 ppm & $1.40 \mathrm{c}$ & 1. $72 a b$ & $1.56 \mathrm{~B}$ & $1.53 \mathrm{~cd}$ & $1.82 \mathrm{a}$ & $1.68 \mathrm{~B}$ \\
\hline Nano Zn 50 ppm & $1.32 \mathrm{c}$ & $1.66 \mathrm{ab}$ & $1.49 \mathrm{~B}$ & $1.45 \mathrm{~cd}$ & $1.74 a b$ & $1.64 \mathrm{~B}$ \\
\hline Nano Zn 100 ppm & $1.63 \mathrm{~b}$ & $1.84 \mathrm{a}$ & $1.74 \mathrm{~A}$ & $1.76 \mathrm{a}$ & $1.91 \mathrm{a}$ & $1.84 \mathrm{~A}$ \\
\hline Mean & $1.33 \mathrm{~B}$ & $1.58 \mathrm{~A}$ & & $1.42 \mathrm{~B}$ & $1.67 \mathrm{~A}$ & \\
\hline
\end{tabular}

Values followed by the same letter (s) are not significantly different at $5 \%$ level

build soil harmfulness. The utilization of nano chemical fertilizers could be an expected way to deal with address such issues of soil harmfulness and other related pressure issues. (Yang et al 2007 and Disfani et al 2017).

\section{2-2 Magnesium (Mg) \%}

Data presented Table (9) suggested that an evident positive effect to both studied factors on $\mathrm{Mg} \%$ of lemongrass plants, but with a different trend. However, irrigation with magnetic water exhibited the higher Mg\% D.W. in both cuts and both studied seasons, compared to those plants irrigated with saline water. All applied treatments recorded higher values of $\mathrm{Mg} \% \mathrm{D} . \mathrm{W}$. than control especially, with 100 and 200 ppm of nano-Mg but 200 ppm was superior in this respect. The increase in $\mathrm{Mg} \%$ with lemongrass plants treated with nanoparticles of $\mathrm{Mg}$ is expected and logic due to the accumulation of $\mathrm{Mg}$ ions in lemongrass plant tissue due to the applied treatments. Interaction values were higher and significant with lemongrass plants irrigated with magnetic water and treated with nano-Mg either at 100 or 200 ppm in both cuts and both studied seasons.

Magnesium (Mg) is essential for the chlorophyll synthesis and contributes up to $10 \%$ of the total $\mathrm{Mg}$ in the chlorophyll structure (Wilkinsan et al 1990). $\mathrm{Mg}^{2+}$ is involved in several vital processes of plants including the formation of ATP in chloroplasts, CO2 stabilization, protein synthesis, chlorophyll formation, development of phloem, and optical oxidation in leaves (Cakmak and Yazici, 2010). Mg is a critical element which is required to maintain a high $\mathrm{pH}$ level in chloroplasts and cytoplasm. According to Wang et al (2004), the concentration of protein and soluble sugars in plant leaves decreases in the $\mathrm{Mg}$ insufficiency condition. Further, Mg2 + deficiency reduces the proline content which has been confirmed by Lesko et al (2002).

\section{4- Fe and Zn (ppm)}

As illustrated in Tables (10 and 11), it is clear that the two studied factors greatly increased $\mathrm{Fe}$ and $\mathrm{Zn}$ levels in lemongrass plants. However, lemongrass plants irrigated with magnetic irrigation water recorded the highest values of $\mathrm{Fe}$ and $\mathrm{Zn}$ in both cuts and both studied season. For instance, lemongrass plants irrigated with magnetic water contained 306.0 and 23.9 ppm of $\mathrm{Fe}$ and $\mathrm{Zn}$, respectively against 276.6 and 20.7 ppm for saline water treatment. The effective treatments in increasing $\mathrm{Fe}$ and $\mathrm{Zn}$ contents were nano-Zn at 100 and 200 ppm in both cuts and both seasons. Interaction between the two studied factors was significant in most cases, but high interaction values for $\mathrm{Fe}$ and $\mathrm{Zn}$ were recorded by lemongrass plants irrigated with magnetic water and treated with 50 and $100 \mathrm{pm}$ of nano-Zn. 

magnesium Treatment of lemongrass plant (Cymbopogen citratus $L$.)

Table 9. Effect of magnetic water, nanoparticles of $\mathrm{Mg}$ and $\mathrm{Zn}$ and their interactions on $\mathrm{Mg} \%$ D.W.of lemongrass (Cymbopogon citratus L.) plant, during 2018/2019 and 2019/2020 seasons.

\begin{tabular}{|c|c|c|c|c|c|c|}
\hline \multirow{3}{*}{ Treatments } & \multicolumn{6}{|c|}{$\begin{array}{c}\text { Mg \% ( D.W.) } \\
\text { 2018/2019 Season }\end{array}$} \\
\hline & \multicolumn{2}{|c|}{$1^{\text {st }}$ cut } & \multirow[b]{2}{*}{ Mean } & \multicolumn{2}{|c|}{$2^{\text {nd }}$ cut } & \multirow[b]{2}{*}{ Mean } \\
\hline & $\begin{array}{l}\text { Saline } \\
\text { water }\end{array}$ & $\begin{array}{c}\text { Magnetic } \\
\text { water }\end{array}$ & & $\begin{array}{l}\text { Saline } \\
\text { water }\end{array}$ & $\begin{array}{c}\text { Magnetic } \\
\text { water }\end{array}$ & \\
\hline Control & $0.32 \mathrm{c}$ & $0.40 \mathrm{bc}$ & $0.36 \mathrm{C}$ & $0.24 \mathrm{e}$ & $0.32 \mathrm{e}$ & $0.28 \mathrm{C}$ \\
\hline Nano Mg 100 ppm & $0.45 a b$ & $0.53 a$ & $0.49 \mathrm{~A}$ & $0.46 \mathrm{~cd}$ & $0.57 \mathrm{ab}$ & $0.52 \mathrm{~B}$ \\
\hline Nano Mg 200 ppm & $0.47 a b$ & $0.55 \mathrm{a}$ & $0.51 \mathrm{~A}$ & $0.48 \mathrm{bcd}$ & $0.63 \mathrm{a}$ & $0.56 \mathrm{~A}$ \\
\hline Nano Zn 50 ppm & $0.44 \mathrm{~b}$ & $0.48 a b$ & $0.46 \mathrm{AB}$ & $0.41 \mathrm{~d}$ & $0.52 \mathrm{bc}$ & $0.47 \mathrm{~B}$ \\
\hline Nano Zn 100 ppm & $0.42 \mathrm{~b}$ & $0.46 \mathrm{ab}$ & $0.44 \mathrm{~B}$ & $0.42 \mathrm{~d}$ & $0.54 \mathrm{abc}$ & $0.48 \mathrm{~B}$ \\
\hline \multirow[t]{2}{*}{ Mean } & $0.42 \mathrm{~B}$ & $0.48 \mathrm{~A}$ & & $0.40 \mathrm{~B}$ & $0.52 \mathrm{~A}$ & \\
\hline & \multicolumn{6}{|c|}{ 2019/2020 Season } \\
\hline Control & $0.37 \mathrm{~d}$ & $0.43 \mathrm{~cd}$ & $0.40 \mathrm{~B}$ & $0.42 \mathrm{~d}$ & $0.46 \mathrm{~cd}$ & $0.44 \mathrm{D}$ \\
\hline Nano Mg 100 ppm & $0.45 \mathrm{bcd}$ & $0.53 a b$ & $0.49 \mathrm{AB}$ & $0.49 \mathrm{bc}$ & $0.63 \mathrm{a}$ & $0.56 \mathrm{AB}$ \\
\hline Nano Mg 200 ppm & $0.50 \mathrm{abc}$ & $0.56 \mathrm{a}$ & $0.53 \mathrm{~A}$ & $0.53 \mathrm{bc}$ & $0.68 \mathrm{a}$ & $0.61 \mathrm{~A}$ \\
\hline Nano Zn 50 ppm & $0.41 \mathrm{~cd}$ & $0.50 a b$ & $0.46 \mathrm{~B}$ & $0.44 \mathrm{~cd}$ & $0.58 a b$ & $0.51 \mathrm{C}$ \\
\hline Nano Zn 100 ppm & $0.42 \mathrm{~cd}$ & $0.55 \mathrm{a}$ & $0.49 \mathrm{AB}$ & $0.48 \mathrm{~cd}$ & $0.58 \mathrm{ab}$ & $0.53 \mathrm{~B}$ \\
\hline Mean & $0.43 \mathrm{~B}$ & $0.51 \mathrm{~A}$ & & $0.47 \mathrm{~B}$ & $0.59 \mathrm{~A}$ & \\
\hline
\end{tabular}

Values followed by the same letter (s) are not significantly different at $5 \%$ level

Table 10. Effect of magnetic water, nanoparticles of $\mathrm{Mg}$ and $\mathrm{Zn}$ and their interactions on Fe (ppm) of lemongrass (Cymbopogon citratus L.) plant, during 2018/2019 and 2019/2020 seasons.

\begin{tabular}{|c|c|c|c|c|c|c|}
\hline \multirow{3}{*}{ Treatments } & \multicolumn{6}{|c|}{$\begin{array}{c}\text { Fe (ppm) } \\
2018 / 2019 \text { Season }\end{array}$} \\
\hline & \multicolumn{2}{|c|}{$1^{\text {st }}$ cut } & \multirow[b]{2}{*}{ Mean } & \multicolumn{2}{|c|}{$2^{\text {nd }}$ cut } & \multirow[b]{2}{*}{ Mean } \\
\hline & $\begin{array}{l}\text { Saline } \\
\text { water }\end{array}$ & $\begin{array}{c}\text { Magnetic } \\
\text { water }\end{array}$ & & $\begin{array}{l}\text { Saline } \\
\text { water }\end{array}$ & $\begin{array}{c}\text { Magnetic } \\
\text { water }\end{array}$ & \\
\hline Control & 200 e & $221 \mathrm{e}$ & $210.5 \mathrm{D}$ & $193 \mathrm{f}$ & 220 ef & $206.5 \mathrm{E}$ \\
\hline Nano Mg 100 ppm & $253 d$ & $261 d$ & $257.0 \mathrm{C}$ & $237 \mathrm{e}$ & $276 d$ & $256.5 \mathrm{D}$ \\
\hline Nano Mg 200 ppm & $302 \mathrm{c}$ & $340 a b$ & $321.0 \mathrm{~B}$ & $281 d$ & $352 b$ & $316.5 \mathrm{C}$ \\
\hline Nano Zn 50 ppm & $312 b c$ & $352 \mathrm{a}$ & $332.0 \mathrm{~A}$ & 337 bc & $388 \mathrm{a}$ & $362.5 \mathrm{~A}$ \\
\hline Nano Zn 100 ppm & $316 \mathrm{bc}$ & $356 \mathrm{a}$ & $336.0 \mathrm{~A}$ & $314 c$ & $362 a b$ & $338.0 \mathrm{~B}$ \\
\hline \multirow[t]{2}{*}{ Mean } & $276.6 \mathrm{~B}$ & $306.0 \mathrm{~A}$ & & $372.4 \mathrm{~B}$ & $319.6 \mathrm{~A}$ & \\
\hline & \multicolumn{6}{|c|}{ 2019/2020 Season } \\
\hline Control & $219 \mathrm{e}$ & $257 \mathrm{~cd}$ & $238.0 \mathrm{D}$ & $201 \mathrm{f}$ & $256 \mathrm{e}$ & $228.5 \mathrm{D}$ \\
\hline Nano Mg 100 ppm & $233 \mathrm{de}$ & $300 \mathrm{~b}$ & $266.5 \mathrm{C}$ & $247 \mathrm{e}$ & $260 \mathrm{e}$ & $253.5 \mathrm{C}$ \\
\hline Nano Mg 200 ppm & $270 \mathrm{c}$ & $302 a b$ & $286.0 \mathrm{~B}$ & $300 \mathrm{~d}$ & $364 a$ & $332.0 \mathrm{~B}$ \\
\hline Nano Zn 50 ppm & $254 \mathrm{~cd}$ & $320 a b$ & $287.0 \mathrm{~B}$ & $332 \mathrm{bc}$ & $357 a b$ & $344.5 \mathrm{~A}$ \\
\hline Nano Zn 100 ppm & $268 \mathrm{c}$ & $332 \mathrm{a}$ & $300.0 \mathrm{~A}$ & $312 \mathrm{~cd}$ & $382 \mathrm{a}$ & $347.0 \mathrm{~A}$ \\
\hline Mean & $248.8 \mathrm{~B}$ & $302.2 \mathrm{~A}$ & & $278.4 \mathrm{~B}$ & $323.8 \mathrm{~A}$ & \\
\hline
\end{tabular}

Values followed by the same letter (s) are not significantly different at $5 \%$ level 
Table 11. Effect of magnetic water, nanoparticles of $\mathrm{Mg}$ and $\mathrm{Zn}$ and their interactions on $\mathrm{Zn}$ (ppm) of lemongrass (Cymbopogon citratus L.) plant, during 2018/2019 and 2019/2020 seasons.

\begin{tabular}{|c|c|c|c|c|c|c|}
\hline \multirow{3}{*}{ Treatments } & \multicolumn{6}{|c|}{$\begin{array}{c}\text { Zn (ppm) } \\
\text { 2018/2019 Season }\end{array}$} \\
\hline & \multicolumn{2}{|c|}{$1^{\text {st }}$ cut } & \multirow[b]{2}{*}{ Mean } & \multicolumn{2}{|c|}{$2^{\text {nd }}$ cut } & \multirow[b]{2}{*}{ Mean } \\
\hline & $\begin{array}{l}\text { Saline } \\
\text { water }\end{array}$ & $\begin{array}{c}\text { Magnetic } \\
\text { water }\end{array}$ & & $\begin{array}{l}\text { Saline } \\
\text { water }\end{array}$ & $\begin{array}{c}\text { Magnetic } \\
\text { water }\end{array}$ & \\
\hline Control & $16.2 \mathrm{f}$ & $19.8 \mathrm{e}$ & $18.0 \mathrm{D}$ & $15.3 \mathrm{f}$ & $20.6 \mathrm{e}$ & $18.0 \mathrm{D}$ \\
\hline Nano Mg 100 ppm & $20.6 \mathrm{e}$ & $22.6 \mathrm{~cd}$ & $21.6 \mathrm{C}$ & $22.1 \mathrm{de}$ & $23.6 \mathrm{bcd}$ & $22.9 \mathrm{C}$ \\
\hline Nano Mg 200 ppm & $21.7 \mathrm{~cd}$ & $23.2 \mathrm{~cd}$ & $22.5 \mathrm{BC}$ & $23.2 \mathrm{~cd}$ & $25.6 \mathrm{~b}$ & $24.4 \mathrm{~B}$ \\
\hline Nano Zn 50 ppm & 21.2 de & $25.8 \mathrm{~b}$ & $23.2 \mathrm{~B}$ & $25.2 \mathrm{~b}$ & $27.6 \mathrm{a}$ & $26.4 \mathrm{~A}$ \\
\hline Nano Zn 100 ppm & $23.7 \mathrm{c}$ & $28.2 \mathrm{a}$ & $26.0 \mathrm{~A}$ & $24.2 \mathrm{bc}$ & $28.9 \mathrm{a}$ & $26.6 \mathrm{~A}$ \\
\hline \multirow[t]{2}{*}{ Mean } & $20.7 \mathrm{~B}$ & $23.9 \mathrm{~A}$ & & $22.0 \mathrm{~B}$ & $25.3 \mathrm{~A}$ & \\
\hline & \multicolumn{6}{|c|}{ 2019/2020 Season } \\
\hline Control & $16.3 \mathrm{f}$ & $21.3 \mathrm{e}$ & $18.8 \mathrm{D}$ & $20.6 \mathrm{e}$ & $23.2 \mathrm{e}$ & $21.9 \mathrm{D}$ \\
\hline Nano Mg 100 ppm & $23.7 \mathrm{de}$ & $27.8 \mathrm{abc}$ & $25.8 \mathrm{C}$ & $22.8 \mathrm{e}$ & $28.7 \mathrm{~cd}$ & $28.5 \mathrm{C}$ \\
\hline Nano Mg 200 ppm & $24.3 d$ & $26.2 \mathrm{bcd}$ & $25.3 \mathrm{C}$ & $26.1 d$ & $34.2 \mathrm{a}$ & $31.2 \mathrm{~B}$ \\
\hline Nano Zn 50 ppm & $25.6 \mathrm{~cd}$ & $28.8 a b$ & $27.2 \mathrm{~B}$ & $27.7 \mathrm{~cd}$ & $32.6 \mathrm{ab}$ & $30.2 \mathrm{~B}$ \\
\hline Nano Zn 100 ppm & $27.7 \mathrm{abc}$ & $30.6 \mathrm{a}$ & $29.2 \mathrm{~A}$ & $30.3 \mathrm{bc}$ & $35.5 \mathrm{a}$ & $32.9 \mathrm{~A}$ \\
\hline Mean & $23.5 \mathrm{~B}$ & $26.9 \mathrm{~A}$ & & $25.5 \mathrm{~B}$ & $30.8 \mathrm{~A}$ & \\
\hline
\end{tabular}

Values followed by the same letter (s) are not significantly different at $5 \%$ level

\section{2-3 Total chlorophyll (meter reading values)}

Table (12) data declared that lemongrass plants irrigated with magnetic water exhibited higher values SPAD of total chlorophyll than those irrigated with saline water in both cuts and both seasons. However, nano-Mg treatments at 100 and 200 ppm were effective than control or nano-Zn of the two used concentrations in recording higher values of total chlorophyll in the two cuts of both seasons. The treatment of nano-Zn at 100 ppm was also effective in some case in increasing total chlorophyll values. The combined treatments of the two studied factors was significant in affecting total chlorophyll of lemongrass plants, where the highest interaction values were recorded by lemongrass plants irrigated with magnetic water and sprayed with 200 ppm of nano-Mg in the two cuts and both studied seasons.

Saltiness stress causes negative effects on different biochemical and physiological procedures which are related with plant development and yield as photosynthesis, protein amalgamation and lipid digestion systems which in this manner are seriously influenced by saltiness disorder inside a plant (Parida and Das 2005). Plant development is truly influenced by salt pressure, and plants adjust to this abiotic condition, Through embracing a modificated systems (Jha et al 2010 and Shabala and Munns, 2012).

Magnetically treated water (MTW), increases the ability of soil to exchange ions and produced good conditions for absorbance of fertilizers by plants. Use of MTW attracts special attention due to its lack of pollution, safety and simplicity, and it may create suitable condition environmentally and good practical application (Bogatin, 1999).

\section{2-4 Proline amino acid content (ug/g d.wt)}

It is clear from data in Table (13) that proline amino acid content was significantly increased in lemongrass plants irrigated with saline water compared with magnetic water treatment. It is well known that the accumulation of proline in a many group of plants due to different types of stresses (Szabados and Savoure 2010). The levels of proline considered a good indicator and vary between species and reached to 100 times higher with water disturbance in comparison with well-watered conditions (Verbruggen and Hermans, 2008). 

magnesium Treatment of lemongrass plant (Cymbopogen citratus $L$.)

Table 12. Effect of magnetic water, nanoparticles of $\mathrm{Mg}$ and $\mathrm{Zn}$ and their interactions on total chlorophyll, SPAD (meter reading) of lemongrass (Cymbopogon citratus L.) plant, during 2018/2019 and 2019/2020 seasons.

\begin{tabular}{|c|c|c|c|c|c|c|}
\hline \multirow{3}{*}{ Treatments } & \multicolumn{6}{|c|}{$\begin{array}{l}\text { Total chlorophyll , SPAD (meter reading) } \\
\text { 2018/2019 Season }\end{array}$} \\
\hline & \multicolumn{2}{|c|}{$1^{\text {st }}$ cut } & \multirow[b]{2}{*}{ Mean } & \multicolumn{2}{|c|}{$2^{\text {nd }}$ cut } & \multirow[b]{2}{*}{ Mean } \\
\hline & $\begin{array}{l}\text { Saline } \\
\text { water }\end{array}$ & $\begin{array}{c}\text { Magnetic } \\
\text { water }\end{array}$ & & $\begin{array}{c}\text { Saline } \\
\text { water }\end{array}$ & $\begin{array}{c}\text { Magnetic } \\
\text { water }\end{array}$ & \\
\hline Control & $22.3 \mathrm{e}$ & $23.1 \mathrm{e}$ & $22.7 \mathrm{C}$ & $21.4 \mathrm{f}$ & $22.3 \mathrm{f}$ & $21.9 \mathrm{D}$ \\
\hline Nano Mg 100 ppm & $25.7 \mathrm{~cd}$ & $26.7 \mathrm{bc}$ & 26.2 B & $25.9 \mathrm{~d}$ & $30.0 \mathrm{ab}$ & $27.8 \mathrm{~B}$ \\
\hline Nano Mg 200 ppm & $26.8 \mathrm{bc}$ & $30.2 \mathrm{a}$ & $28.4 \mathrm{~A}$ & $28.1 \mathrm{bc}$ & $31.9 \mathrm{a}$ & $30.0 \mathrm{~A}$ \\
\hline Nano Zn 50 ppm & $24.1 \mathrm{de}$ & $26.3 c$ & $25.2 \mathrm{~B}$ & $23.2 \mathrm{e}$ & $24.4 \mathrm{de}$ & $23.8 \mathrm{C}$ \\
\hline Nano Zn 100 ppm & $27.2 \mathrm{bc}$ & $28.9 \mathrm{ab}$ & $28.1 \mathrm{~A}$ & $26.8 \mathrm{c}$ & $28.1 \mathrm{bc}$ & $27.5 \mathrm{~B}$ \\
\hline \multirow[t]{2}{*}{ Mean } & $25.5 \mathrm{~B}$ & $27.0 \mathrm{~A}$ & & $25.1 \mathrm{~B}$ & $27.3 \mathrm{~A}$ & \\
\hline & \multicolumn{6}{|c|}{ 2019/2020 Season } \\
\hline Control & $20.6 \mathrm{f}$ & $22.8 \mathrm{e}$ & $21.7 \mathrm{D}$ & $20.5 \mathrm{e}$ & $23.1 \mathrm{~cd}$ & $21.8 \mathrm{E}$ \\
\hline Nano Mg 100 ppm & $25.0 \mathrm{~cd}$ & $27.5 \mathrm{~b}$ & 26.3 B & $23.1 \mathrm{c}$ & $24.6 \mathrm{bc}$ & $23.9 \mathrm{C}$ \\
\hline Nano Mg 200 ppm & 27.7 b & $29.8 \mathrm{a}$ & $28.8 \mathrm{~A}$ & $26.2 \mathrm{~b}$ & $30.0 \mathrm{a}$ & $28.1 \mathrm{~A}$ \\
\hline Nano Zn 50 ppm & $23.4 \mathrm{de}$ & $26.5 \mathrm{bc}$ & $25.0 \mathrm{C}$ & $21.9 \mathrm{de}$ & $26.5 b$ & $24.2 \mathrm{C}$ \\
\hline Nano Zn 100 ppm & $24.7 \mathrm{~cd}$ & $27.4 \mathrm{~b}$ & $26.1 \mathrm{~B}$ & $22.4 \mathrm{~d}$ & $31.2 \mathrm{a}$ & $26.8 \mathrm{~B}$ \\
\hline Mean & $24.3 \mathrm{~B}$ & $26.7 \mathrm{~A}$ & & $22.8 \mathrm{~B}$ & $27.1 \mathrm{~A}$ & \\
\hline
\end{tabular}

Values followed by the same letter (s) are not significantly different at $5 \%$ level

Table 13. Effect of magnetic water, nanoparticles of $\mathrm{Mg}$ and $\mathrm{Zn}$ and their interactions on proline amino acid content (Ug/g D.wt) of lemongrass (Cymbopogon citratus L.) plant, during 2018/2019 and 2019/2020 seasons.

\begin{tabular}{|c|c|c|c|c|c|c|}
\hline \multirow{3}{*}{ Treatments } & \multicolumn{6}{|c|}{$\begin{array}{l}\text { Proline amino acid content ( } \mu \mathrm{g} / \mathrm{g} \text { D.wt) } \\
2018 / 2019 \text { Season }\end{array}$} \\
\hline & \multicolumn{2}{|c|}{$1^{\text {st }}$ cut } & \multirow[b]{2}{*}{ Mean } & \multicolumn{2}{|c|}{$2^{\text {nd }}$ cut } & \multirow[b]{2}{*}{ Mean } \\
\hline & $\begin{array}{l}\text { Saline } \\
\text { water }\end{array}$ & $\begin{array}{l}\text { Magnetic } \\
\text { water }\end{array}$ & & $\begin{array}{l}\text { Saline } \\
\text { water }\end{array}$ & $\begin{array}{c}\text { Mag- } \\
\text { netic wa- } \\
\text { ter }\end{array}$ & \\
\hline Control & $91.7 \mathrm{a}$ & $62.9 \mathrm{e}$ & $77.3 \mathrm{~A}$ & $98.0 \mathrm{a}$ & $72.9 \mathrm{~cd}$ & $85.5 \mathrm{~A}$ \\
\hline Nano Mg 100 ppm & $87.7 \mathrm{a}$ & $60.1 \mathrm{e}$ & $73.9 \mathrm{~B}$ & $82.9 \mathrm{~b}$ & $57.3 \mathrm{~g}$ & $70.1 \mathrm{~B}$ \\
\hline Nano Mg 200 ppm & $78.2 \mathrm{c}$ & $52.3 \mathrm{f}$ & $65.3 \mathrm{C}$ & $67.1 \mathrm{de}$ & 64.8 ef & $66.0 \mathrm{C}$ \\
\hline Nano Zn 50 ppm & $82.7 \mathrm{~b}$ & $58.7 \mathrm{e}$ & 70.7 B & $76.0 \mathrm{c}$ & 63.2 ef & $69.6 \mathrm{~B}$ \\
\hline Nano Zn 100 ppm & $68.9 \mathrm{~d}$ & $47.8 \mathrm{f}$ & $58.4 \mathrm{D}$ & $61.9 \mathrm{f}$ & $53.5 \mathrm{~g}$ & $57.7 \mathrm{D}$ \\
\hline \multirow[t]{2}{*}{ Mean } & $81.8 \mathrm{~A}$ & $56.4 \mathrm{~B}$ & & $77.2 \mathrm{~A}$ & $62.3 \mathrm{~B}$ & \\
\hline & \multicolumn{6}{|c|}{ 2019/2020 Season } \\
\hline Control & $93.3 \mathrm{a}$ & $61.9 \mathrm{~d}$ & $77.6 \mathrm{~A}$ & $91.4 \mathrm{a}$ & $78.0 \mathrm{c}$ & $84.7 \mathrm{~A}$ \\
\hline Nano Mg 100 ppm & $87.0 \mathrm{~b}$ & $56.2 \mathrm{e}$ & $71.6 \mathrm{~B}$ & $82.0 \mathrm{bc}$ & $68.6 \mathrm{~d}$ & $75.6 \mathrm{~B}$ \\
\hline Nano Mg 200 ppm & $85.3 b$ & $56.8 \mathrm{e}$ & $71.1 \mathrm{~B}$ & $84.0 \mathrm{~b}$ & $62.1 \mathrm{e}$ & $73.1 \mathrm{~B}$ \\
\hline Nano Zn 50 ppm & $76.4 \mathrm{c}$ & $60.3 \mathrm{de}$ & $68.4 \mathrm{C}$ & $78.0 \mathrm{c}$ & $59.0 \mathrm{e}$ & $68.5 \mathrm{C}$ \\
\hline Nano Zn 100 ppm & $61.8 \mathrm{~d}$ & $49.7 \mathrm{f}$ & $55.8 \mathrm{D}$ & $68.1 \mathrm{~d}$ & $51.1 \mathrm{f}$ & $59.6 \mathrm{D}$ \\
\hline Mean & $80.8 \mathrm{~A}$ & $57.0 \mathrm{~B}$ & & $80.4 \mathrm{~A}$ & $63.8 \mathrm{~B}$ & \\
\hline
\end{tabular}

Values followed by the same letter (s) are not significantly different at $5 \%$ level 
In the first cut of first season, lemongrass plants irrigated with saline water recorded $81.8 \mu \mathrm{g}$ proline/g d.wt compared to $56.4 \mu \mathrm{g}$ proline/g d.wt for magnetic water irrigated lemongrass plants. However, all applied treatments were effective in reducing proline levels than control with significant differences between them. The least proline values were recorded by $100 \mathrm{ppm}$ nano-Zn treatment in both cuts of both seasons, whereas least interaction values of proline were detected by the lemongrass plants irrigated with magnetic water and sprayed with $100 \mathrm{ppm}$ nano-Zn. It is suggested that proline acts in membrane and protein protection against the effects of the high concentration of inorganic ions and temperature extremes, in the stabilization of cell structures and detoxification of free radicals (Verbruggen and Hermans, 2008) and as a way to store carbon, nitrogen and energy (Hare and Cress, 1997). The reduction of proline amino acid level is an evident indicator to that the plant did not suffers from abiotic or biotic stress due to the applied treatments. Proline accumulation in the leaves of plants exposed to drought stress is not only associated with an increased expression of the some gene but also with the decreased expression of proline dehydrogenase genes $(\mathrm{PDH})$ coding for the enzymes of proline degradation (Miller et al 2009).

\section{2-2 Glycine betaine content ( $\mu \mathrm{g} / \mathrm{g}$ d.wt)}

Table (14) show the effect of both quality of irrigation water and nano particles of $\mathrm{Mg}$ and $\mathrm{Zn}$ spraying on glycine betaine levels of lemongrass plants. Glycine betaine (GB) component is synthesized via two distinct pathways from two distinct substrates, choline and glycine (Ashraf and Foolad, 2007). The osmolyte GB can accumulate in a several organisms such as plants, animals, bacteria, cyanobacteria, and algae (Rhodes and Hanson, 1993); Generally, values of glycine betaine as an indicator for plant stress were registered the lowest values of proline content although both components are considered great indicators for suffering of plants from different types of stress. Lemongrass plants irrigated with saline water exhibited higher values of glycine betaine than those irrigated with magnetic water which explained the relationship between stress and accumulation of glycine betaine component. It is important to mention that accumulation of GB provides protection against several environmental factors such as drought, salinity, and cold (Chen and Murata, 2008). GB is biosynthesized in plants when exposed to diverse environmental factors that cause stress, such as salinity. It has been observed that GB can be synthesized and accumulated; however, some species such as Oryza sativa, Arabidopsis thaliana, and Nicotiana tabacum do not produce GB naturally (Rhodes and Hanson, 1993).

However, the treatment of $100 \mathrm{ppm}$ nano-Zn was more effective in reducing glycine betaine level than other treatments or control in both cuts and both seasons. Interaction values were significant in most cases where the least values of glycine betaine were recorded in lemongrass plants irrigated with magnetic water and sprayed with $100 \mathrm{ppm}$ of nano-Zn. Other explains suggested that osmotic stress-induced GB biosynthesis occurs via jasmonate signal transduction, which not only has a key role in osmotic stress resistance but also contributes to tolerance (Xu et al 2018). Furthermore, GB maintained a higher photosynthesis rate, thereby increasing the production and translocation of sucrose via phloem loading to enhance the plant response to low-phosphate stress ( $\mathrm{Li}$ et al 2019). However, Wei et al (2017) demonstrated that GB might regulate ion channel and transporters, resulting in high potassium and low sodium levels to enhance salt tolerance in transgenic plants under salt stress conditions.

\subsection{Volatile oil content (\%)}

At it shown in Table (15), lemongrass plants irrigated with magnetic water produced higher volatile oil \% than those irrigated with saline water in the two cuts and two studied seasons. However, in first cut in first season, volatile oil \%, of lemongrass plants irrigated with magnetic water was $1.48 \%$ against $0.83 \%$ in saline water irrigated plants. Regarding the effect of the used treatments, it is clear that all applied treatments increased volatile oil \% than control except the treatment of $100 \mathrm{ppm}$ of nano-Mg. The higher volatile oil \% were obtained with 100 ppm of nano-Zn treatments, where it was superior than others in both cuts and both studied seasons. Interaction between the two studied factors was significant in most cases, the highest values in this respect were recorded in the lemongrass plants irrigated with magnetic water and sprayed with 100 ppm of nano-Zn. 

magnesium Treatment of lemongrass plant (Cymbopogen citratus $L$.)

Table 14. Effect of magnetic water, nanoparticles of $\mathrm{Mg}$ and $\mathrm{Zn}$ and their interactions on glycine betaine content (Ug/g D.wt) of lemongrass (Cymbopogon citratus L.) plant, during 2018/2019 and 2019/2020 seasons.

\begin{tabular}{|c|c|c|c|c|c|c|}
\hline \multirow{3}{*}{ Treatments } & \multicolumn{6}{|c|}{$\begin{array}{l}\text { Glycine betaine content }(\mu \mathrm{g} / \mathrm{g} \text { D.wt) } \\
2018 / 2019 \text { Season }\end{array}$} \\
\hline & \multicolumn{2}{|c|}{$1^{\text {st }}$ cut } & \multirow[b]{2}{*}{ Mean } & \multicolumn{2}{|c|}{$2^{\text {nd }}$ cut } & \multirow[b]{2}{*}{ Mean } \\
\hline & $\begin{array}{l}\text { Saline } \\
\text { water }\end{array}$ & $\begin{array}{l}\text { Magnetic } \\
\text { water }\end{array}$ & & $\begin{array}{l}\text { Saline } \\
\text { water }\end{array}$ & $\begin{array}{c}\text { Mag- } \\
\text { netic wa- } \\
\text { ter }\end{array}$ & \\
\hline Control & $41.5 \mathrm{a}$ & $20.5 d$ & $31.0 \mathrm{~A}$ & $42.0 \mathrm{a}$ & $24.0 \mathrm{de}$ & $33.0 \mathrm{~A}$ \\
\hline Nano Mg 100 ppm & 35.2 b & $18.1 \mathrm{de}$ & 26.7 B & $35.5 \mathrm{~b}$ & $23.6 \mathrm{de}$ & 29.6 B \\
\hline Nano Mg 200 ppm & $32.8 \mathrm{bc}$ & $14.9 \mathrm{e}$ & $22.9 \mathrm{CD}$ & $32.3 \mathrm{bc}$ & 22.9 e & $27.6 \mathrm{BC}$ \\
\hline Nano Zn 50 ppm & $30.3 \mathrm{bc}$ & $17.1 \mathrm{de}$ & $23.7 \mathrm{C}$ & $29 . .7 \mathrm{c}$ & $23.4 \mathrm{e}$ & $26.6 \mathrm{C}$ \\
\hline Nano Zn 100 ppm & $27.2 \mathrm{c}$ & $14.7 \mathrm{e}$ & $21.0 \mathrm{D}$ & $28.3 \mathrm{~cd}$ & 20.8 e & $24.6 \mathrm{D}$ \\
\hline \multirow[t]{2}{*}{ Mean } & $34.9 \mathrm{~A}$ & $19.1 \mathrm{~B}$ & & $33.6 \mathrm{~A}$ & $22.9 \mathrm{~B}$ & \\
\hline & \multicolumn{6}{|c|}{ 2019/2020 Season } \\
\hline Control & $39.2 \mathrm{a}$ & $25.1 \mathrm{~cd}$ & $32.2 \mathrm{~A}$ & $43.0 \mathrm{a}$ & $27.7 d$ & $35.4 \mathrm{~A}$ \\
\hline Nano Mg 100 ppm & $36.9 \mathrm{a}$ & $21.2 \mathrm{de}$ & 29.1 B & $38.8 a b$ & $23.1 \mathrm{de}$ & $31.0 \mathrm{~B}$ \\
\hline Nano Mg 200 ppm & 31.7 b & $18.3 \mathrm{e}$ & $25.0 \mathrm{C}$ & $35.3 \mathrm{bc}$ & 20.7 ef & $28.0 \mathrm{C}$ \\
\hline Nano Zn 50 ppm & $29.7 \mathrm{bc}$ & 20.6 de & $25.2 \mathrm{C}$ & $40.0 \mathrm{ab}$ & 19.9 ef & $30.0 \mathrm{~B}$ \\
\hline Nano Zn 100 ppm & $27.8 \mathrm{bc}$ & $17.8 \mathrm{e}$ & $22.8 \mathrm{D}$ & $33.4 \mathrm{c}$ & $17.5 \mathrm{f}$ & $25.5 \mathrm{D}$ \\
\hline Mean & $33.1 \mathrm{~A}$ & $20.6 \mathrm{~B}$ & & $38.1 \mathrm{~A}$ & $21.8 \mathrm{~B}$ & \\
\hline
\end{tabular}

Values followed by the same letter (s) are not significantly different at $5 \%$ level

Table 15. Effect of magnetic water, nanoparticles of $\mathrm{Mg}$ and $\mathrm{Zn}$ and their interactions on volatile oil \% of lemongrass (Cymbopogon citratus L.) plant, during 2018/2019 and 2019/2020 seasons

\begin{tabular}{|c|c|c|c|c|c|c|}
\hline \multirow{3}{*}{ Treatments } & \multicolumn{6}{|c|}{$\begin{array}{c}\text { Volatile oil \% (air dried herb) } \\
2018 / 2019 \text { Season }\end{array}$} \\
\hline & \multicolumn{2}{|c|}{$1^{\text {st }}$ cut } & \multirow[b]{2}{*}{ Mean } & \multicolumn{2}{|c|}{$2^{\text {nd }}$ cut } & \multirow[b]{2}{*}{ Mean } \\
\hline & $\begin{array}{l}\text { Saline } \\
\text { water }\end{array}$ & $\begin{array}{c}\text { Magnetic } \\
\text { water }\end{array}$ & & $\begin{array}{c}\text { Saline } \\
\text { water }\end{array}$ & $\begin{array}{c}\text { Magnetic } \\
\text { water }\end{array}$ & \\
\hline Control & $0.54 \mathrm{f}$ & $0.88 \mathrm{de}$ & $0.71 \mathrm{D}$ & $0.31 \mathrm{f}$ & $0.66 \mathrm{cde}$ & $0.49 \mathrm{D}$ \\
\hline Nano Mg 100 ppm & 0.70 ef & $1.13 \mathrm{c}$ & $0.79 \mathrm{D}$ & $0.58 \mathrm{e}$ & $0.84 \mathrm{c}$ & $0.71 \mathrm{C}$ \\
\hline Nano Mg 200 ppm & $0.91 d$ & $1.82 a b$ & $1.37 \mathrm{~B}$ & $0.79 \mathrm{~cd}$ & $1.21 \mathrm{ab}$ & $1.00 \mathrm{~A}$ \\
\hline Nano Zn 50 ppm & $0.83 \mathrm{de}$ & $1.67 \mathrm{~b}$ & $1.25 \mathrm{C}$ & $0.62 \mathrm{de}$ & $1.06 \mathrm{~b}$ & $0.84 \mathrm{~B}$ \\
\hline Nano Zn 100 ppm & $1.17 \mathrm{c}$ & $1.92 \mathrm{a}$ & $1.55 \mathrm{~A}$ & $0.81 \mathrm{c}$ & $1.35 \mathrm{a}$ & $1.08 \mathrm{~A}$ \\
\hline \multirow[t]{2}{*}{ Mean } & $0.83 \mathrm{~B}$ & $1.48 \mathrm{~A}$ & & $0.62 \mathrm{~B}$ & $1.02 \mathrm{~A}$ & \\
\hline & \multicolumn{6}{|c|}{ 2019/2020 Season } \\
\hline Control & $0.41 \mathrm{e}$ & $0.82 \mathrm{~cd}$ & $0.77 \mathrm{C}$ & $0.45 \mathrm{e}$ & $0.78 \mathrm{~cd}$ & $0.62 \mathrm{C}$ \\
\hline Nano Mg 100 ppm & $0.68 d$ & $0.96 \mathrm{bc}$ & $0.82 \mathrm{C}$ & $0.61 \mathrm{de}$ & $0.83 \mathrm{c}$ & $0.72 \mathrm{C}$ \\
\hline Nano Mg 200 ppm & $0.82 \mathrm{~cd}$ & $1.28 \mathrm{a}$ & $1.05 \mathrm{~B}$ & $0.89 \mathrm{c}$ & $1.36 \mathrm{ab}$ & $1.13 \mathrm{~A}$ \\
\hline Nano Zn 50 ppm & $0.87 \mathrm{bc}$ & $1.05 \mathrm{~b}$ & $0.96 \mathrm{~B}$ & $0.77 \mathrm{~cd}$ & $1.17 \mathrm{~b}$ & $0.97 \mathrm{~B}$ \\
\hline Nano Zn 100 ppm & $0.93 \mathrm{bc}$ & $1.47 \mathrm{a}$ & $1.20 \mathrm{~A}$ & $0.96 \mathrm{c}$ & $1.51 \mathrm{a}$ & $1.24 \mathrm{~A}$ \\
\hline Mean & 0.74 B & $1.12 \mathrm{~A}$ & & $0.74 \mathrm{~B}$ & $1.13 \mathrm{~A}$ & \\
\hline
\end{tabular}

Values followed by the same letter (s) are not significantly different at $5 \%$ level 


\section{REFERENCES}

A.O.A.C. 1990. Official Methods of Analysis Twelfth ed. Published by the association of official analytical chemists.

AbeSato S.Y., Inoue S., Ishibashi H., Maruyama N. and Takizawa T. 2002. Anti-Candida albicans activity of essential oils including lemongrass (Cymbopogon citratus) oil and its component, Citral. Jap J. Med Mycol.; 44, 285291.

Almutairi Z.M. 2016. Effect of nano-silicon application on the expression of salt tolerance genes in germinating tomato (Solanum lycopersicum L.) seedlings under salt stress. Plant. Omics 9 106-114.

Ashraf M. and Foolad M.R. 2007. Roles of glycine betaine and proline in improving plant abiotic resistance. Environ. Exp. Bot. 59, 206-216.

Bagaturiya N.S. 1990. Lemongrass essential oil. Pishch Prom-st. (Moscow). 10, 48 p.

Bates L.S., Waldren R.P. and Teare I.D. 1973. Rapid determination of free proline for water stress studies. Plant Soil 39(1), 205-207.

Bogatin J. 1999. Magnetic treatment of irrigation water: experimental results and application conditions. Environmental Sci. \& Technology, 33(8), 1280-1285.

Bringham F.T. 1982. Methods of Soil Analysis, (Ed), Part 2, Agronomy. 9, 431-447.

British Pharmacopoeia 1963. In "Determination of Volatile Oil in Drugs". The Pharmaceutical Press, London.

Cakmak I. and Yazici A.M. 2010. Magnesium: a forgotten element in crop production. Better Crops 94, 23-25.

Chapman H.D. and Pratt P.R. 1982. Analysis for Soils, Plants, and Waters. Pub- Search 69, 237 277.

Chen J., Zhang H., Zhang X. and Tang M. 2017. Arbuscular mycorrhizal symbiosis alleviates salt stress in black locust through improved photosynthesis, water status, and $\mathrm{K}+/ \mathrm{Na}+640$ homeostasis. Front. Plant Sci., 8, 1739.

Chen T.H. and Murata N. 2008. Glycine betaine: An effective protectant against abiotic stress in plants. Trends Plant Sci. 13, 499-505.

Ciaramello D. 1973. Preliminary study of the use of citronella, lemongrass, palmarosa and vetiver for cellulose and paper production. Biol. Tech. (Inst. Agronomy, Campinas). 1, 24 p. Campinas, Brazil.
Deng B.O. and Feng P.X. 2007. Variations of optic properties of water under action of static magnetic field, Chinese Sci. Bulletin. 52(23), 31793182.

Disfani M.N., Mikhak A., Kassaee M.Z. and Maghari A. 2017. Effects of nano $\mathrm{Fe} / \mathrm{SiO} 2$ fertilizers on germination and growth of barley and maize. Arch. Agron. Soil Sci., 63, 817-826.

EL-Metwally A.E., Abdalla F.E., EL-Saady A.M., Safina S.A. and EL-Sawy S.S. 2010.' Response of wheat to magnesium and copper foliar feeding under sandy soil condition'. J. of American Sci., 6(12), 818-823.

Escalante-Magana C., Aguilar-Caamal F., Echevarria-Machado I., Fatima Medina-Lara L. Cach S. and Martinez-Esteve M. 2019. Contribution of glycine betaine and proline to water deficit tolerance in pepper plants. Hortsceince: 54(6), 1044-1054.

Fatma M., Masood A., Per T.S., Rasheed F. and Khan N.A. 2016. Interplay between nitric oxide and sulfur assimilation in salt tolerance in plants. Crop J., 4, 153-161.

Flores M., Carbonell M.V. and Martinez E. 2007. Exposure of maize seeds to stationary magnetic fields: Effects on germination and early growth. Environmental and Experimental Botany, 59, 68-75.

Flowers T.J. 2004 "Improving crop salt tolerance," J. Experimental Botany, 55(396), 307-319.

Guebel D.V., Nudel B.C., Giuletti A.M. 1991. Simple and rapid micro-kjeldahl method for total nitrogen analysis. Biotechnol. Tech. 5(6), 427430.

Hare P.D. and Cress W.A. 1997. Metabolic implications of stress-induced proline accumulation in plants. Plant Growth Regulation 21(2), 79102.

Hawrylak-Nowak B., Matraszek R. and Szyma M. 2010. Selenium modifies the effect of short-term chilling stress on cucumber plants. Biol. Trace Element. Res. 138, 307-315.

Hilal M.H. and Hillal M.M. 2000. Application of magnetic technologies in desert agriculture .1Seed germination and seedling emergence of some crop in a saline calcareous soil. Egypt $\mathbf{J}$. Soil Sci., 40(3), 413-421.

Jha D., Shirley N., Tester M. and Roy S.J. 2010. Variation in salinity tolerance and shoot sodium accumulation in Arabidopsis ecotypes linked to differences in the natural expression levels of transporters involved in sodium transport. Plant Cell Environ. 33, 793-804. 

magnesium Treatment of lemongrass plant (Cymbopogen citratus $L$.)

Kumar R., Sarawgi A.K., Ramos C., Amarante S. T., Ismaeil A.M. and Wade L. J. 2006. 'Partitioning of dry matter during drought stress in rainfed low land rice'. Field Crop Research, 98(1), 1-11.

Kwak J.I. and An Y.J. 2016. The current state of the art in research on engineered nanomaterials and terrestrial environments: Different scale approaches. Environ. Res. 151, 368-382.

Lesko K., Banyai E.S. and Sarkadi L.S. 2002. 'Effect of magnesium on free amino acid and polyamine content in wheat seedling exposed to cadmium stress'. Acta Biologica Szegediensis, 46(34), 109-111.

Li D., Zhang T., Wang M. Liu Y., Brestic M., Chen T.H. and Yang X. 2019. Genetic engineering of the biosynthesis of glycine betaine modulates phosphate homeostasis by regulating phosphate acquisition in tomato. Front. Plant Sci. 9, 1-13.

Mahajan P., Dhoke S. and Khanna A. 2011. Effect of nano-ZnO particle suspension on growth of mung (Vigna radiata) and gram (Cicer arietinum) seedlings using plant agar method. J. Nanotech., Article ID 696535.7p.

Maitwijczuk A., Kornarzyski K. and Pietruszewski S. 2012. Effect of magnetic field on seed germination and seedling growth of sunflower. International Agrophysics, 26(3), 271278.

Miller G., Honig A., Stein H., Suzuki N., Mittler R., Zilberstein A. 2009. Unraveling delta1-pyrroline-5-carboxylate-proline cycle in plants by uncoupled expression of proline oxidation enzymes. J. Biol. Chem. 284, 26482-26492.

Munns R., and Tester M. 2008. "Mechanisms of salinity tolerance," Annual Review of Plant Biology, 59, 651-681.

Nair R.S., Varghese H., Nair B.G., Maekawa T., Yoshida Y. and Kumar D.S. 2010. Nanoparticulate material delivery to plants. Plant Sci., 179, 154-163.

Noran R., Shani R. and Lin I. 1996. The effect of irrigation with magnetically treated water on the translocation of minerals in the soil. Magn. Electr. Sep., 7, 109-122.

Pang X.F. and Deng B. 2008. Investigation of changes in properties of water under the action of a magnetic field. Sci. China Ser. G: Phys. Mech. Astro. 51, 1621-1632.

Parida A.K. and Das A.B. 2005. "Salt tolerance and salinity effect on plants: a review," Ecotoxicol. Environ. Saf., 60, 324-349.
Pohl P., Dzimitrowicz A., Jedryczko D., Szymczycha-Madeja A., Welna M. and Jamroz P. 2016. The determination of elements in herbal teas and medicinal plant formulations and their tisanes. J. Pharm. Biomed. Anal. 130, 326-335.

Prasad T.N., Sudhakar P., Sreenivasulu Y., Latha P., Munaswamy V., Reddy K.R., Sreeprasad T.S. and Pradeep T. 2012. Effect of nanoscale zinc oxide particles on the germination, growth and yield of peanut. J. Plant. Nutr., 35, 905-927.

Rhodes D. and Hanson A.D. 1993. Quaternary ammonium and tertiary sulfonium compounds in higher plants. Anni. Rev. Plant Physiol and Plant Mol. Biol. 44(1), 357-384.

Roghayyeh S., Sedghi M., Shishevan M.T. and Sharifi R.S. 2010. Effects of Nano-Iron Oxide Particles on Agronomic Traits of Soybean. Not Sci. Biol. 2(2), 112-113.

Shabala S. and Munns R. 2012. Salinity stress: Physiological constraints and adaptive mechanisms. In Plant Stress Physiology; Shabala, S., Ed.; CABI: Wallingford, UK, pp. 59-93.

Singh A., Singh N., Hussain I., Singh H., Yadav V. and Singh S. 2016. Green synthesis of nano zinc oxide and evaluation of its impact on germination and metabolic activity of Solanum lycopersicum. J. Biotechnol. 233, 84-94.

Snedecor G.W. and Cochran W.G. 1990. Statistical Methods. $11^{\text {th }} \mathrm{Ed}$. lowa State College Press. Ames, lowa, U.S.A. pp. 369-373.

Sturikova H., Krystofova O., Huska D. and Adam V. 2018. Zinc, zinc nanoparticles and plants. J. Hazard. Mater., 349, 101-110.

Szabados L. and Savoure A. 2010. Proline: A multifunctional amino acid. Trends Plant Sci. 15(2), 89-97.

Tarafdar J.C. and Claassen N. 2003. Organic phosphorus utilization by wheat plants under sterilized condition. Biol Fert Soils 39, 25-29.

Tiwari M., Dwivedi U.N. and Kakkar P. 2010. Suppression of oxidative stress and pro-inflammatory mediators by Cymbopogon citratus DC. Stapf extract in lipopolysaccharide stimulated murine alveolar macrophages. Food Chem. Toxicol. 10(48), 2913-2919.

Tyagi A.K. and Malik A. 2012. Morphostructural damage in food-spoiling bacteria due to the Lemon grass oil and its vapor: SEM, TEM, and AFM investigations. Biology, Medicine Published in Evidence-Based Complementary Alternat Med. 
Verbruggen N. and Hermans C. 2008. Proline accumulation in plants: A review. Amino Acids 35(4), 753-759.

Verma S.S. 2011. Magnetic water treatment. Chem. Bus. J. 1, 13-16.

Waleed A.J., Riyadh A.H. and Hussein F.H. 2013. Effect of magnetic field on seed germination of Triticum aestivum. World J. of Agric. Sci., 1, 168-171.

Waller R.A. and Duncan D.B. 1969. A Bayes rule for the symmetric multiple comparisons problem. J. of the American Statistical Association, 64(328), 1484-1503.

Wang F., Liu P. and Zhu J. 2004. 'Effect of magnesium $(\mathrm{Mg})$ on contents of free proline, soluble sugar and protein in soybean leaves'. Henan Agricultural Sciences, 6, 35-38.

Wei D., Zhang W., Wang C., Meng Q., Li G., Chen T.H., and Yang X. 2017. Genetic engineering of the biosynthesis of glycine betaine leads to alleviate salt-induced potassium efflux and enhances salt tolerance in tomato plants. Plant Sci. 257, 74-83.

Westerman R.L. 1990. Soil Testing and Plant Analysis. ( $3^{\text {rd }}$ ed.) Soil Science Society of America, Inc. Madison Wisconsin, USA.
Wilkinson S., Welch R., Mayland H. and Grunes D. 1990. Magnesium in plants: uptake, distribution, function and utilization by man and animals. Metal lons Biol Syst 26, 33-56.

Xu Z., Sun M., Jiang X., Sun H., Dang X., Cong H. and Qiao F. 2018. Glycine betaine biosynthesis in response to osmotic stress depends on jasmonate signaling in watermelon suspension cells. Front. Plant Sci. 9, 1469.

Xu Z.Z., Zhou G.S. and Shimizu H. 2009. Effects of soil drought with nocturnal warming on leaf stomatal traits and mesophyll cell ultrastructure of a perennial grass. Crop. Sci., 49, 1843-1851.

Yang F., Liu C., Gao F., Su M., Wu X., Zheng L., Hong F. and Yang P. 2007. The improvement of spinach growth by nano-anatase $\mathrm{TiO}_{2}$ treatment is related to nitrogen photoreduction. Biol. Trace Elem. Res. 119, 77-88.

Yang R.L., Du H.W., Lin Z.Q., Yang L.L., Zhu H., Zhang H., Tang Z.K. and Gui X.C. 2019. ZnO nanoparticles filled tetrapod-shaped carbon shell for lithium-sulfur batteries. Carbon, 141, 258-265.

Zaimenko N.V., Didyk N.P., Dzyuba O.I., Zakrasov O.V., Rositska N.V., Viter A.V. 2014. Enhancement of drought resistance in wheat and corn by nanoparticles of natural mineral analcite. Ecologia Balkanica 6, 1-10. 


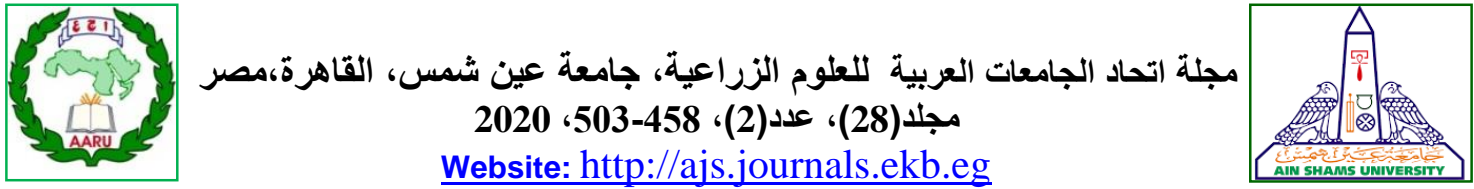

\section{تجنب الاجهاد الملحى من خلال المعاملة بالماء الممغنط والنانو زنكك وإلنانو ماغنسيوم في نبات حشيشة الليمون}

[35]

\author{
أحمد نظمي عبد الحميد" \\ قسم البساتين - كلية الزراعة - جامعة عين شمس- ص.ب 68 - حدائق شبرا 11241 - القاهرة - مصر \\ *Corresponding author: nazmy604@yahoo.com
}

Received 15 July, 2020

Accepted 4 August, 2020

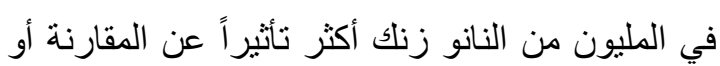

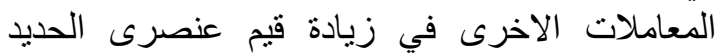

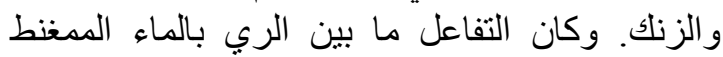

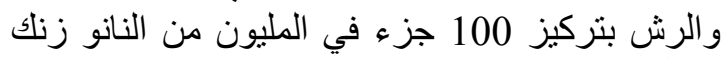

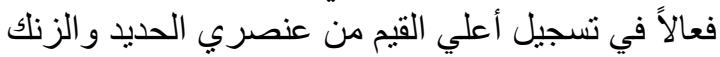

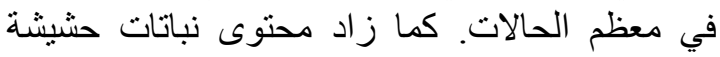

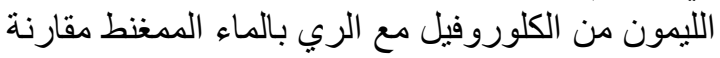

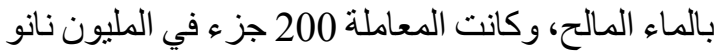

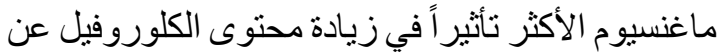

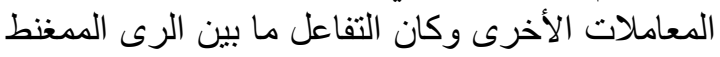

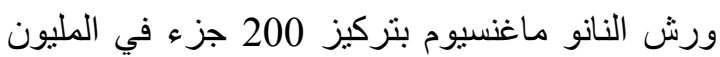

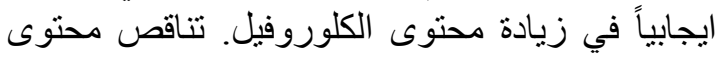
نباتات حشيشة الليمون من الحمض الئرئ الاميني البرولين،

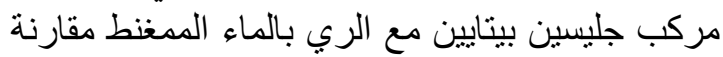

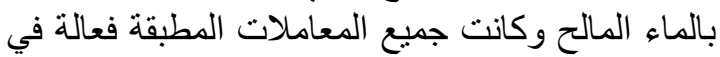
تقليل محتوي النباتات من كلا المركبين كمؤشر جيد الماتيد

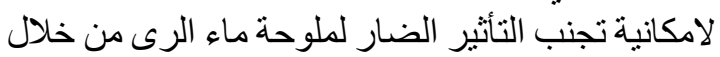

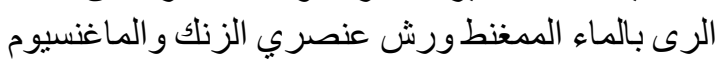

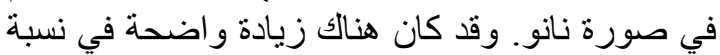

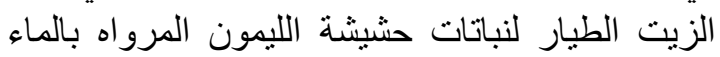

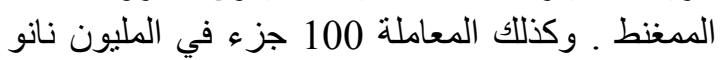

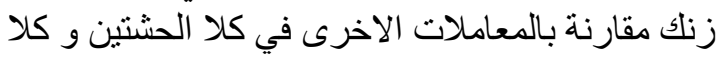

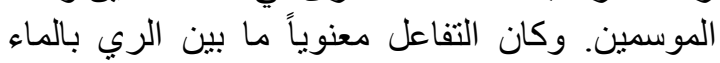
الممغنط وتطبيق المعاملات المذكورة في تحسين نسبة لئن

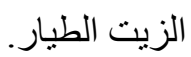

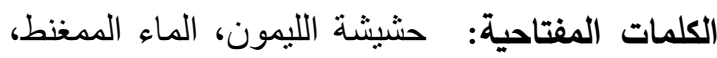

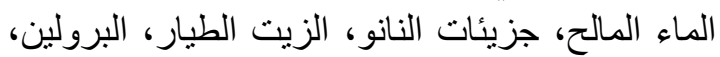

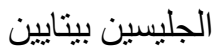

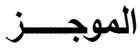

درس نأثير كلاً من الماء الممغنط مقارنة بالماء

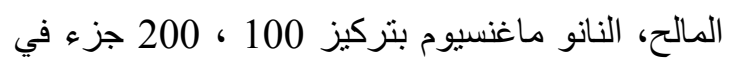
المليون، النانو زنك بتركيز 50، 100 جزء في في المليون

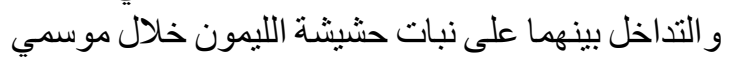
2018 / 2019 ، 2019 / 2020 ـ و وقد تأثرت قيات فياسات

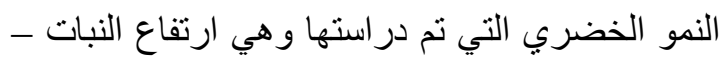

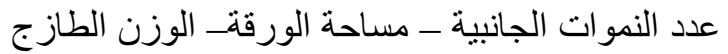

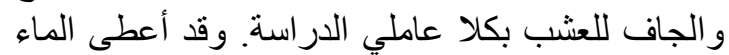

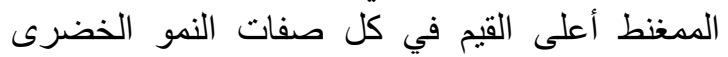

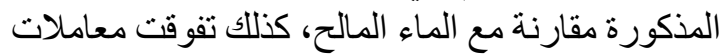

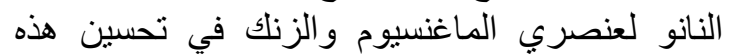

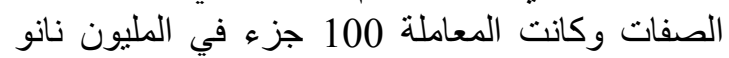

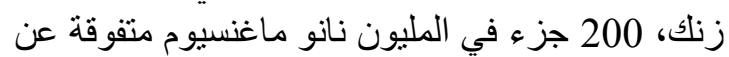

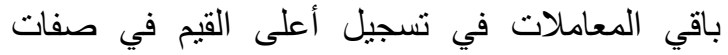

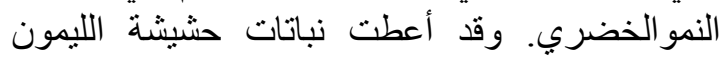

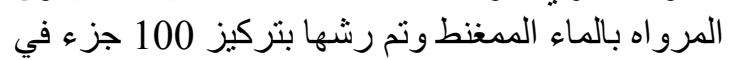

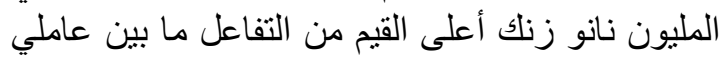

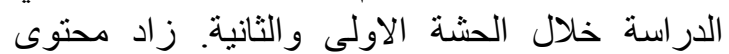

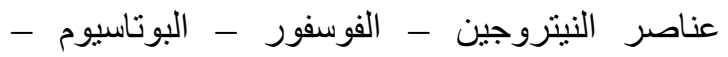

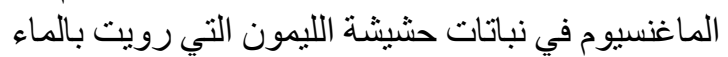

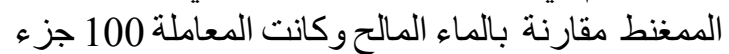

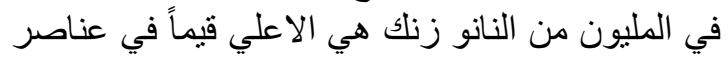

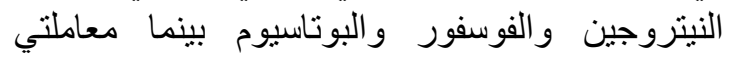
الماغنسيوم 100، 200 جزء في في المليون كانت متفوقة

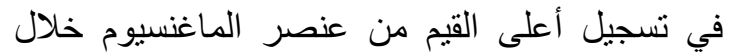

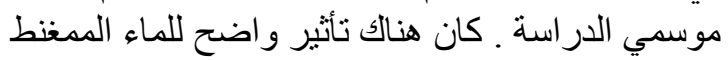

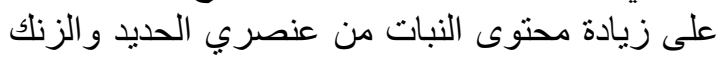
مقارنة بالماء المالح، وكانت المعاملة 50 ، 100 جزء الماء

تحكيم: 1.د أحمد عبدالعال حجازي محمد

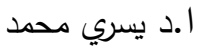

